\title{
Dislocation dynamics in hexagonal close-packed crystals
}

\author{
S. Aubry, M. Rhee, G. Hommes, V.V Bulatov, and A. Arsenlis \\ Lawrence Livermore National Laboratory, \\ P.O. Box 808, Livermore, CA 94551.
}

\begin{abstract}
Extensions of the dislocation dynamics methodology necessary to enable accurate simulations of crystal plasticity in hexagonal close-packed (HCP) metals are presented. They concern the introduction of dislocation motion in HCP crystals through linear and non-linear mobility laws, as well as the treatment of composite dislocation physics. Formation, stability and dissociation of $<\mathrm{c}+\mathrm{a}>$ and other dislocations with large Burgers vectors defined as composite dislocations are examined and a new topological operation is proposed to enable their dissociation. The results of our simulations suggest that composite dislocations are omnipresent and may play important roles both in specific dislocation mechanisms and in bulk crystal plasticity in HCP materials. While fully microscopic, our bulk DD simulations provide wealth of data that can be used to develop and parameterize constitutive models of crystal plasticity at the mesoscale.
\end{abstract}

Key words: dislocation dynamics, hexagonal close-packed, composites dislocations.

\section{Introduction}

Metals and alloys with a hexagonal close-packed (HCP) crystal structure exhibit considerable variations in the mechanical and physical properties essential for their technological applications. For instance, magnesium's low density and high specific strength make its alloys attractive as potential candidates for replacing steels and other high-density materials in the automotive industry [1]. Titanium is being used in the aerospace and in the medical industries for its strength to weight and corrosion characteristics [2]. Beryllium has been considered for nuclear fuel cladding, nuclear fuel compacts, and as a neutron moderator for fission power plants dating back to the early 1950's. However, the highly anisotropic mechanical properties and, in particular, limited ductility of beryllium and magnesium alloys have thus far restrained their practical 
uses. Efforts to improve the technologically relevant properties of HCP materials may be aided by developing a mechanistic understanding of their straining response at different length scales: from discrete atoms to macroscopic continuum. The dislocation dynamics (DD) method has been developed [3] to evaluate the plastic behavior of materials at the mesoscopic scale, and to determine the strength of body-centered cubic (BCC) metals [4] as an integrated part of a multiscale approach to develop such a mechanistic understanding of BCC strength. This multiscale approach can be extended to determine the strength of HCP metals like beryllium, titanium, and magnesium.

The plastic response of HCP materials is a complex interplay between dislocation slip and twinning. Depending on the material and straining conditions, one of the two mechanisms may dominate the other. In particular, the propensity to slip on $<\mathrm{c}+\mathrm{a}>$ systems and the competition with twinning is thought to be critical for deformation aligned with the c axis. A number of twinning modes have been observed and linked to enhanced ductility of HCP materials, as reviewed in [5-10] and references therein. In this paper, we will focus solely on dislocation plasticity in HCP crystals through the dislocation dynamics (DD) method. The mesoscale physics of twinning, while important, is considered to be outside the scope of this paper. Our focus on the discrete dislocation plasticity of HCP crystals lays the groundwork for considering the more complex nature of dislocation slip plus twinning in plastic deformation at the mesoscale in future studies.

To the authors' knowledge, three large-scale dislocation dynamics studies have been concerned with HCP crystals. Monnet et al. [11] studied prismatic slip in zirconium using a 3D discrete dislocation dynamics capability. They focused their study on dislocations interacting in different prismatic planes and proposed mobility laws for screw and non-screw segments in a phenomenological manner for these slip systems. Capolungo et al. [12] showed the weak effect of anisotropic calculations in magnesium. More recently, Bertin et al. [13] have used DD in magnesium crystals to determine latent hardening parameters for different slip systems. All three of the aforementioned studies did not include in their formulation any special treatment of $<\mathrm{c}+\mathrm{a}>$ dislocations. They assumed that $<\mathrm{c}+\mathrm{a}>$ dislocations and other dislocations with large Burgers vector magnitude were elastically stable and did not consider their possible instability.

Low ductility in magnesium and its inability to achieve high plastic strains have been linked to the existence of $<\mathrm{c}+\mathrm{a}>$ dislocations in HCP crystals $[14,15]$. The importance of the stability of these large Burgers vector dislocations has been extensively studied over the last few decades $[16,17,14,18,15]$. In [16], Yoo et al. analyzed the formation of $\langle\mathrm{c}+\mathrm{a}\rangle$ dislocations from an attractive junction between $\langle\mathrm{c}\rangle$ and $\langle\mathrm{a}\rangle$ dislocations and Yoo [17] showed that at room temperature, the pure edge $<\mathrm{c}+\mathrm{a}>$ dislocation in the $\{11 \overline{2} 2\}$ glide planes are 
unstable and rather stable in pure screw orientation using isotropic elasticity for different HCP crystals. These results have been further examined through experimental techniques such as transmission electron microscopy(TEM) $[14,18]$. Tonda and Ando [18] showed experimentally that the dissociation of $\langle\mathrm{c}+\mathrm{a}\rangle$ edge dislocation into a $\langle\mathrm{c}>$ sessile and an $<\mathrm{a}>$ glissile edge dislocation can happen in a thermally activated process. Agnew et al [14] also studied in details formation and dissociation of $<\mathrm{c}+\mathrm{a}>$ dislocations using TEM in compressed magnesium along the $c$ axis as well as magnesium-lithium alloys. They observed evidence of several types of dissociation of $<\mathrm{c}+\mathrm{a}>$ dislocations, some of them involving $\langle\mathrm{c}\rangle,\langle\mathrm{a}\rangle$, and partial dislocations. Recent molecular dynamics combined with density functional theory simulations [15] has linked $<\mathrm{c}+\mathrm{a}>$ dislocations dissociation with low ductility in magnesium. Given the evidence that the stability of $\langle\mathrm{c}+\mathrm{a}\rangle$ dislocations and other large Burgers vectors that may develop during the plastic slip deformation of HCP crystals may be finite, it warrants a DD study to illuminate how the finite stability of such dislocations would affect our understanding of HCP plasticity at the mesoscopic scale.

This paper is organized as follows. In Section 2, the enhancements to the ParaDiS code [3] for the simulation of HCP crystals are described in detail. Subsection 2.1 describes the implementation of HCP slip geometries. Subsection 2.2 describes the development of mobility functions for that geometry. The framework of the mobility law is such that it is capable of incorporating experimental measurements or MD simulation results on the mobility of dislocations with specific orientations and interpolating between them. Subsections 2.3-2.5 detail the physics of composite dislocations in HCP crystals, the topological operations invoked for their dissociation, and the decision tree associated with the dissociation of composite dislocations and the cross-slip of screw dislocations, respectively. In Section 3, numerical simulations are conducted with the enhanced version of ParadiS starting with small scale simulations of unstable $<\mathrm{c}+\mathrm{a}>$ dislocations in Subsection 3.1 and binary junctions of $<\mathrm{c}+\mathrm{a}>$ dislocations in Subsections 3.2 and 3.3. Finally, a few large scale simulations of the deformation of bulk single crystal Be are conducted to illustrate novel features in the microstructural evolution of HCP crystals in Subsection 3.4. In these simulations, the new topological operation and mobility laws are exercised and a detailed description of the evolution of the dislocations densities and fluxes per slip system is determined. This coarse-grained information will be used in a subsequent paper to construct a crystal plasticity model for HCP materials. A discussion of the results and set of concluding remarks may be found in Section 4. 


\section{Implementation of dislocation dynamics for HCP materials}

\subsection{HCP slip crystallography}

The geometry of a hexagonal close-packed lattice is fully defined by two lattice parameters $a$ and $c$. The commonly used notation for crystallographic planes and directions due to Damiano [19] is intended to emphasize the symmetry of the HCP lattice by using Miller-Bravais indices [20] referring to four axes $\boldsymbol{a}_{1}, \boldsymbol{a}_{2}, \boldsymbol{a}_{3}$ and $\boldsymbol{c}$, as shown in Fig. 1. For numerical practicality in our DD implementation we express all lattice vectors and planes in a Cartesian frame that converts to the standard Miller-Bravais representation as follows (Fig. 1):

$$
\begin{aligned}
& \boldsymbol{a}_{1}=-\frac{1}{2} a \boldsymbol{e}_{\boldsymbol{x}}+\frac{\sqrt{3}}{2} a \boldsymbol{e}_{\boldsymbol{y}} \\
& \boldsymbol{a}_{2}=-\frac{1}{2} a \boldsymbol{e}_{\boldsymbol{x}}-\frac{\sqrt{3}}{2} a \boldsymbol{e}_{\boldsymbol{y}} \\
& \boldsymbol{a}_{3}=a \boldsymbol{e}_{\boldsymbol{x}} \\
& \boldsymbol{a}_{4}=c \boldsymbol{e}_{\boldsymbol{z}}
\end{aligned}
$$

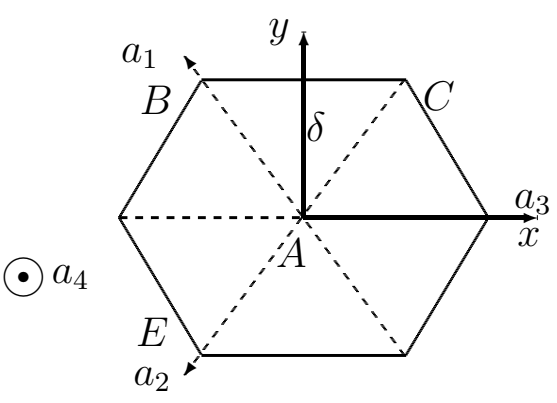

(a)

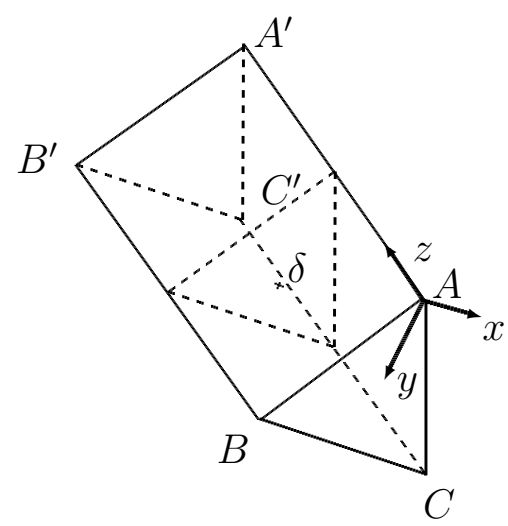

(b)

Fig. 1. Damiano's notation for the HCP lattice [19]: $A$ is at the origin, $\left(\boldsymbol{a}_{\mathbf{1}}, \boldsymbol{a}_{\mathbf{2}}\right.$, $\left.\boldsymbol{a}_{\mathbf{3}}\right)$ are three vectors in the basal plane with respect to which the first three Miller indices are defined. The fourth Miller index, $\boldsymbol{a}_{\mathbf{4}}$, defines the component orthogonal to the basal plane.

Tables 1- 3 list all the slip systems defined in our DD model as shown in Fig. 1[b]. Three types of Burgers vectors are considered, $\langle\mathrm{a}\rangle,\langle\mathrm{c}\rangle$ and $<\mathrm{a}+\mathrm{c}>$ with lengths $a, c$ and $\sqrt{a^{2}+c^{2}}$, respectively. The (0001) basal plane is the only plane of its kind, whereas there are three symmetry-related but geometrically distinct prismatic planes of the $<10 \overline{1} 0>$ type, six primary pyramidal planes of $<10 \overline{1} 1>$ type and six secondary pyramidal planes of $<11 \overline{2} 2>$ type. In the following, we refer to each specific slip system by a 
Table 1

$\mathrm{HCP}$ slip systems for the Burgers vectors of type $<\mathrm{a}>$. Each set of Burgers vector and glide plane is given a specific notation. For instance, the first Burgers vector in the basal plane is noted 1Ba.

$\begin{array}{llll}\text { Burgers vector } & \text { Planes } & \text { Plane type } & \text { Notation } \\ \boldsymbol{b}_{1}=1 / 3[2 \overline{1} \overline{1} 0] & (0001) & \text { basal } & 1 \mathrm{Ba} \\ & (01 \overline{1} 0) & \text { prismatic } & 1 \mathrm{Pr} \\ & (01 \overline{1} 1) & \text { pyramidal } & 1 \mathrm{PyI} \\ & (0 \overline{1} 11) & \text { pyramidal } & 1 \mathrm{PyII} \\ & & & \\ \boldsymbol{b}_{2}=1 / 3[\overline{1} \overline{1} 20] & (0001) & \text { basal } & 2 \mathrm{Ba} \\ & (1 \overline{1} 00) & \text { prismatic } & 2 \mathrm{Pr} \\ & (1 \overline{1} 01) & \text { pyramidal } & 2 \mathrm{PyI} \\ & (\overline{1} 101) & \text { pyramidal } & 2 \mathrm{PyII} \\ & & & \\ \boldsymbol{b}_{3}=1 / 3[\overline{1} 2 \overline{1} 0] & (0001) & \text { basal } & 3 \mathrm{Ba} \\ & (\overline{1} 010) & \text { prismatic } & 3 \mathrm{Pr} \\ & (\overline{1} 011) & \text { pyramidal } & 3 \mathrm{PyI} \\ & (10 \overline{1} 1) & \text { pyramidal } & 3 \mathrm{PyII}\end{array}$

unique notation in which the first numeral refers to one of ten glissile Burgers vectors, followed by a letter-Roman numeral notation for the glide plane. The Burgers vectors numbered 1 through 3 are of type $<\mathrm{a}>$, the Burgers vectors 4 through 9 are of type $\left\langle\mathrm{c}+\mathrm{a}>\right.$, and the $10^{\text {th }}$ Burgers vector is of type $<\mathrm{c}>$. The glide plane that a dislocation belongs to is indicated by an abbreviation of the plane's name: Ba stands for the basal plane; Pr stands for a prismatic plane; PyI represents the first of two primary pyramidal planes; and PyII represents the second pyramidal plane of the same primary type. sP stands for the secondary pyramidal plane in which only the $\langle\mathrm{c}+\mathrm{a}>$ dislocations can glide. As an example, 3Pr in Table 1 refers to the Burgers vector $\boldsymbol{b}_{\mathbf{3}}$ of type $<\mathrm{a}>$ in the prismatic plane (1010). Refer to Tables 1- 3 and Fig. 1 for all notations. In this paper, only dislocations that are contained within this family of slip systems will be considered glissile. Dislocations that appear as a result of reactions that do not belong to one of these systems will be considered sessile. The mobility of these two dislocation classes is discussed in the next section. 
Table 2

HCP slip systems for the Burgers vectors of type $<\mathrm{c}+\mathrm{a}>$ (continued)

$\begin{array}{llll}\text { Burgers vector } & \text { Planes } & \text { Plane type } & \text { Notation } \\ \boldsymbol{b}_{4}=1 / 3[2 \overline{1} \overline{1} 3] & (01 \overline{1} 0) & \text { prismatic } & 4 \mathrm{Pr} \\ & (\overline{1} 011) & \text { pyramidal } & 4 \mathrm{PyI} \\ & (\overline{1} 101) & \text { pyramidal } & 4 \mathrm{PyII} \\ & (\overline{2} 112) & \text { second pyramidal } & 4 \mathrm{sP}\end{array}$

$$
\begin{array}{llll}
\boldsymbol{b}_{5}=1 / 3[2 \overline{1} \overline{1} \overline{3}] & (01 \overline{1} 0) & \text { prismatic } & 5 \mathrm{Pr} \\
& (10 \overline{1} 1) & \text { pyramidal } & 5 \mathrm{PyI} \\
& (1 \overline{1} 01) & \text { pyramidal } & 5 \mathrm{PyII} \\
& (2 \overline{1} \overline{1} 2) & \text { second pyramidal } & 5 \mathrm{sP}
\end{array}
$$

$$
\begin{array}{llll}
\boldsymbol{b}_{6}=1 / 3[\overline{1} \overline{1} 23] & (1 \overline{1} 00) & \text { prismatic } & 6 \mathrm{Pr} \\
& (10 \overline{1} 1) & \text { pyramidal } & 6 \mathrm{PyI} \\
& (01 \overline{1} 1) & \text { pyramidal } & 6 \mathrm{PyII} \\
& (11 \overline{2} 2) & \text { second pyramidal } & 6 \mathrm{sP}
\end{array}
$$

$$
\begin{array}{llll}
\boldsymbol{b}_{7}=1 / 3[\overline{1} \overline{1} 2 \overline{3}] & (1 \overline{1} 00) & \text { prismatic } & 7 \mathrm{Pr} \\
& (\overline{1} 011) & \text { pyramidal } & 7 \mathrm{PyI} \\
& (0 \overline{1} 11) & \text { pyramidal } & 7 \mathrm{PyII} \\
& (\overline{1} \overline{1} 22) & \text { second pyramidal } & 7 \mathrm{sP}
\end{array}
$$

$$
\begin{array}{llll}
\boldsymbol{b}_{8}=1 / 3[\overline{1} 2 \overline{1} 3] & (\overline{1} 010) & \text { prismatic } & 8 \mathrm{Pr} \\
& (1 \overline{1} 01) & \text { pyramidal } & 8 \mathrm{PyI} \\
& (0 \overline{1} 11) & \text { pyramidal } & 8 \mathrm{PyII} \\
& (1 \overline{2} 12) & \text { second pyramidal } & 8 \mathrm{sP}
\end{array}
$$

$$
\begin{array}{llll}
\boldsymbol{b}_{9}=1 / 3[\overline{1} 2 \overline{1} \overline{3}] & (\overline{1} 101) & \text { prismatic } & 9 \mathrm{Pr} \\
& (01 \overline{1} 1) & \text { pyramidal } & 9 \mathrm{PyI} \\
& (\overline{1} 101) & \text { pyramidal } & 9 \mathrm{PyII} \\
& (\overline{1} 2 \overline{1} 2) & \text { second pyramidal } & 9 \mathrm{sP}
\end{array}
$$


Table 3

HCP slip systems for the Burgers vectors of type $<c>$ (continued)

$\begin{array}{llll}\text { Burgers vector } & \text { Planes } & \text { Plane type } & \text { Notation } \\ \boldsymbol{b}_{10}=\left[\begin{array}{llll}0 & 0 & 0 & 1\end{array}\right] & (01 \overline{1} 0) & \text { prismatic } & 10 \operatorname{PrI} \\ & (\overline{1} 010) & \text { prismatic } & 10 \operatorname{PrII} \\ & (1 \overline{1} 00) & \text { prismatic } & 10 \operatorname{PrIII}\end{array}$

\subsection{Treatment of dislocation motion}

Methods for computing forces on dislocation segments and nodes have been detailed in $[3,21]$ for isotropic elasticity and in $[22,23]$ for anisotropic elasticity. The response of dislocation segments and nodes to these forces is defined by dislocation mobility functions that may depend on local stress state, pressure, and temperature as well as on the dislocation's geometry: Burgers vector, glide plane, segment orientation, etc. Although, in general, dislocation equations of motion are non-linear, simpler linear mobility laws have been proposed for BCC and face-centered cubic (FCC) crystals in [3] and [24], respectively.

Molecular Dynamics (MD) simulations of dislocation motion are increasingly being used to determine dislocation mobility functions $[25,26]$. They typically output dislocation velocities that are either non-linear or linear functions of stress for specific dislocation line directions, whereas DD simulations require the mobility of a dislocation to be defined for any arbitrary line direction. This section describes a method for constructing a mobility function for dislocation dynamics simulations for an arbitrary line direction given information about the mobility of dislocations in particular line directions. A simple linear version of this mobility function is employed in the DD simulations of an HCP single crystal conducted in this paper.

Assuming that dislocation inertia can be neglected, the equation of motion of a point on a dislocation segment takes the following form

$$
\boldsymbol{v}(\boldsymbol{x})=\mathcal{M}(\boldsymbol{F}(\boldsymbol{x})
$$

where $\boldsymbol{v}(\boldsymbol{x})$ is the velocity of a differential dislocation segment at point $\boldsymbol{x}$ on the dislocation line and $\mathcal{M}$ is a generally non-linear vector function of vector argument $\boldsymbol{F}(\boldsymbol{x})$ which is the force per unit length on the same differential segment. Assuming further that mobility $\mathcal{M}$ is a monotonic single-valued function of $\boldsymbol{F}(\boldsymbol{x})$, it is useful to define its inverse drag function $\mathcal{B}$ which generally depends on segment orientation and other parameters

$$
\boldsymbol{F}(\boldsymbol{x})=\mathcal{M}^{-1}(\boldsymbol{v}(\boldsymbol{x}))=\mathcal{B}(\boldsymbol{v}(\boldsymbol{x}))
$$


ParaDiS [3] is a nodal dislocation dynamics model in which curved dislocation lines are discretized into nodes connected by straight segments, and only the forces and velocities on the nodes are explicitly considered. Within such an approximation, local velocity $\boldsymbol{v}(\boldsymbol{x})$ cannot vary independently along the line segments and thus can not exactly satisfy equation of motion Eq. 2 at every point $\boldsymbol{x}$ on the dislocation line. The force and velocity can be matched in a weak sense (approximately) at the nodes, e.g.

$$
\boldsymbol{F}_{i}+\boldsymbol{F}_{i}^{\mathrm{drag}}=0
$$

where

$$
\boldsymbol{F}_{i}=\sum_{j \text { neighbor of } i} \int_{\boldsymbol{x}_{i}}^{\boldsymbol{x}_{j}} N_{i j}(\boldsymbol{x}) \boldsymbol{F}(\boldsymbol{x}) d \ell(\boldsymbol{x})
$$

and

$$
\boldsymbol{F}_{i}^{\text {drag }}=-\sum_{j \text { neighbor of } i} \int_{\boldsymbol{x}_{i}}^{\boldsymbol{x}_{j}} N_{i j}(\boldsymbol{x}) \mathcal{B}(v(\boldsymbol{x})) d \ell(\boldsymbol{x}) .
$$

$\boldsymbol{F}_{i}$ is the total thermo-mechanical force on dislocation node $\boldsymbol{x}_{i}$, and $\boldsymbol{F}_{i}^{\mathrm{drag}}$ is the drag force exerted on node $\boldsymbol{x}_{i} . N_{i j}(\boldsymbol{x})=\frac{\left|\boldsymbol{x}-\boldsymbol{x}_{j}\right|}{\left|\boldsymbol{x}_{i}-\boldsymbol{x}_{j}\right|}$ is the shape function (integration weight) for the line segment connecting two neighboring nodes at positions $\boldsymbol{x}_{\boldsymbol{i}}$ and $\boldsymbol{x}_{\boldsymbol{j}}$, and the integrals are taken over all segments $\left[\boldsymbol{x}_{i}, \boldsymbol{x}_{j}\right]$ connected to node $\boldsymbol{x}_{i}$.

In a finely discretized dislocation network, one can assume that local velocity $\boldsymbol{v}(\boldsymbol{x})$ at every point along segment $\left[\boldsymbol{x}_{i}, \boldsymbol{x}_{j}\right]$ is approximately the same as the velocity at node $\boldsymbol{x}_{i}$

$$
\boldsymbol{v}_{i j}(\boldsymbol{x}) \approx \boldsymbol{v}_{i}
$$

simplifying the integral expression, Eq. 5 into

$$
\boldsymbol{F}_{i}^{\mathrm{drag}}=-\frac{1}{2} \sum_{j \text { neighbor of } i} \mathcal{B}_{i j}\left(\boldsymbol{v}_{i}\right)\left|\boldsymbol{L}_{i j}\right|
$$

where $\boldsymbol{L}_{i j}=\boldsymbol{x}_{j}-\boldsymbol{x}_{i}$ is the segment vector with end nodes $\boldsymbol{x}_{i}$ and $\boldsymbol{x}_{j}$.

In principle, MD simulations can supply mobility (or drag) functions for any point $\boldsymbol{x}$ on a dislocation line taking into account the glide and climb of dislocations of arbitrary orientation (character). However, in practice, MD dislocation mobility data is available only for a limited set of dislocation characters and straining conditions. Commonly, mobility simulations are usually conducted only for pure edge and pure screw dislocations in a few specific slip planes 
(see for example [1] and [25]). Interpolation and extrapolation must be used to complete a dislocation mobility function for DD simulations over the rest of orientation space where no MD data is available.

When the drag functions are known for the pure screw with line direction $\boldsymbol{t}$ and two pure edges with line directions $\boldsymbol{e}_{1}$ and $\boldsymbol{e}_{2}$, a drag function at node $\boldsymbol{x}_{i}$ for an arbitrary segment character $\left[\boldsymbol{x}_{i}, \boldsymbol{x}_{j}\right]$ may be interpolated in the form

$$
\mathcal{B}_{i j}\left(\boldsymbol{v}_{i}\right)=\frac{\left|L_{i j}^{s}\right|}{\left|\boldsymbol{L}_{i j}\right|} \mathcal{B}_{i j}^{s}\left(\boldsymbol{v}_{i}\right)+\frac{\left|L_{i j}^{e_{1}}\right|}{\left|\boldsymbol{L}_{i j}\right|} \mathcal{B}_{i j}^{e_{1}}\left(\boldsymbol{v}_{i}\right)+\frac{\left|L_{i j}^{e_{2}}\right|}{\left|\boldsymbol{L}_{i j}\right|} \mathcal{B}_{i j}^{e_{2}}\left(\boldsymbol{v}_{i}\right),
$$

where $L_{i j}^{s}, L_{i j}^{e_{1}}$, and $L_{i j}^{e_{2}}$ are the components of segment vector $\boldsymbol{L}_{i j}$ expressed in the basis $\left(\boldsymbol{t}, \boldsymbol{e}_{1}, \boldsymbol{e}_{2}\right)$, i.e.

$$
\boldsymbol{L}_{i j}=L_{i j}^{s} \boldsymbol{t}+L_{i j}^{e_{1}} \boldsymbol{e}_{\mathbf{1}}+L_{i j}^{e_{2}} \boldsymbol{e}_{\mathbf{2}}
$$

Interpolation procedures appropriate for cases when mobility (drag) parameters are available for more than three line orientations are discussed in Appendix A.

Combining Eq. 7 and Eq. 8, we obtain

$$
\boldsymbol{F}_{i}^{\mathrm{drag}}=-\frac{1}{2} \sum_{j \text { neighbor of } i}\left|L_{i j}^{s}\right| \mathcal{B}_{i j}^{s}\left(\boldsymbol{v}_{i}\right)+\left|L_{i j}^{e_{1}}\right| \mathcal{B}_{i j}^{e_{1}}\left(\boldsymbol{v}_{i}\right)+\left|L_{i j}^{e_{2}}\right| \mathcal{B}_{i j}^{e_{2}}\left(\boldsymbol{v}_{i}\right) .
$$

The velocity $\boldsymbol{v}_{\boldsymbol{i}}$ of node $\boldsymbol{x}_{i}$ can be obtained by solving Eq. 3 .

In a number of materials and straining conditions, such as FCC metals strained at room temperature, linear functions provide sufficiently accurate descriptions of dislocation mobility. In other cases, linear mobility is assumed for lack of data and/or for computational expedience. In DD simulations of HCP beryllium presented in this paper a linear mobility equation

$$
\mathcal{B}_{i j}\left(\boldsymbol{v}_{i}\right)=\boldsymbol{B}_{i j} \boldsymbol{v}_{i}
$$

is adopted resulting in the following expression for the nodal drag force

$$
\begin{array}{r}
\boldsymbol{F}_{i}^{\mathrm{drag}}=-\frac{1}{2} \sum_{j \text { neighbor of } i}\left(\left|L_{i j}^{s}\right| \boldsymbol{B}_{i j}^{s}(\boldsymbol{b}, \boldsymbol{n})+\left|L_{i j}^{e_{1}}\right| \boldsymbol{B}_{i j}^{e_{1}}(\boldsymbol{t}, \boldsymbol{b})+\right. \\
\left.\left|L_{i j}^{e_{2}}\right| \boldsymbol{B}_{i j}^{e_{2}}(\boldsymbol{t}, \boldsymbol{b})+\left|L_{i j}^{\mathrm{jct}}\right| \boldsymbol{B}_{i j}^{\mathrm{jct}}(\boldsymbol{t})\right) \boldsymbol{v}_{i} .
\end{array}
$$

The last term on the right hand side is introduced specifically to account for a very low (nearly zero) mobility of sessile dislocation segments that do not 
belong to any of the slip systems defined in Tables 1- 3. Since most such segments result from collisions of mobile dislocation lines, we refer to all such dislocations as "junctions". For such segments, $L_{i j}^{j c t}=L_{i j}$ while all the other components of the segment vector decomposition entering Eq. 12 are set to zero.

The various components of the segment drag tensors entering Eq. 12 are defined as follows

$$
\boldsymbol{B}^{s}(\boldsymbol{b}, \boldsymbol{n})=B^{\text {line }}(\boldsymbol{b}, \boldsymbol{n}) \boldsymbol{t}_{s} \otimes \boldsymbol{t}_{s}+B_{s}^{\mathrm{glide}}(\boldsymbol{b}, \boldsymbol{n}) \boldsymbol{m}_{s} \otimes \boldsymbol{m}_{s}+B_{s}^{\mathrm{climb}}(\boldsymbol{b}, \boldsymbol{n}) \boldsymbol{n} \otimes \boldsymbol{n},
$$

where $\boldsymbol{t}_{s}=\frac{\boldsymbol{b}}{|\boldsymbol{b}|}$ is the screw line direction, $\boldsymbol{n}$ is the unit normal of a predefined glide plane, and $\boldsymbol{m}_{s}=\boldsymbol{t}_{s} \times \boldsymbol{n}$. The $B_{s}^{\text {glide }}$ and $B_{s}^{\text {climb }}$ drag coefficients can be obtained from MD simulations or experiments, and the fictitious drag coefficient, $B^{\text {line }}$, defines the resistance to numerical node redistribution along the line. For the edge segments

$$
\boldsymbol{B}^{e}\left(\boldsymbol{t}_{e}, \boldsymbol{b}_{e}, \boldsymbol{n}\right)=B^{\text {line }}(\boldsymbol{b}, \boldsymbol{n}) \boldsymbol{t}_{e} \otimes \boldsymbol{t}_{e}+B_{e}^{\mathrm{glide}}(\boldsymbol{b}, \boldsymbol{n}) \boldsymbol{m}_{e} \otimes \boldsymbol{m}_{e}+B_{e}^{\mathrm{climb}}(\boldsymbol{b}, \boldsymbol{n}) \boldsymbol{n}_{e} \otimes \boldsymbol{n}_{e}
$$

where the line direction is either $\boldsymbol{t}_{\boldsymbol{e}}=\boldsymbol{e}_{1}$ or $\boldsymbol{t}_{e}=\boldsymbol{e}_{\mathbf{2}}$, one of the edge directions used in the line segment decomposition, Eq. $9 ; \boldsymbol{m}_{\boldsymbol{e}}=\frac{\boldsymbol{b}}{\mid \boldsymbol{b} b}$, and $\boldsymbol{n}_{\boldsymbol{e}}=\boldsymbol{m}_{e} \times \boldsymbol{t}_{e}$. The drag coefficients for the edge drag matrix have the same meaning as for the screw drag matrix. These drag parameters depend on each segment's slip system (pure screw dislocation segments, along with edges, are assigned glide planes in this particular screw drag model). Finally, for junction segments

$$
\boldsymbol{B}^{\text {jct }}(\boldsymbol{t})=B^{\text {sessile }}(\boldsymbol{I}-\boldsymbol{t} \otimes \boldsymbol{t})+B^{\text {jct line }} \boldsymbol{t} \otimes \boldsymbol{t}
$$

where $\boldsymbol{t}$ is the segment's direction vector and $\boldsymbol{I}$ is the identity matrix. In this last expression, coefficient $B^{\text {sessile }}$ is chosen to be large but finite, to facilitate stable inversion of the nodal drag matrix in Eq. 12 used to solve for the nodal velocity in simulations focused on the dislocation slip plasticity of $\mathrm{HCP}$ crystals.

To illustrate the linear mobility law presented in this section, the drag parameters used for beryllium throughout this paper are given in Table 4. In Table 4, some drag parameters are based on [27], the other coefficients were determined so that the ratio between the drag coefficients of the different slip systems were representative of the literature for HCP crystals. Drag parameters for line and climb directions were chosen to be respectively softer and stiffer than the drag parameter of screw dislocations in the prismatic plane. Molecular dynamics simulations can be used to determine these coefficients more precisely. However, dislocation drag parameters for dislocations on slip systems with $<\mathrm{c}>$ and $<\mathrm{c}+\mathrm{a}>$ Burgers vectors have proven difficult to obtain. Molecular dynamics and density functional theory techniques often used to determine these coefficients are out of the scope of this paper. In [1], some 
drag parameters are given for magnesium hcp crystals. Techniques to obtain these coefficients are explained in [26].

Table 4

Drag coefficients for beryllium hcp crystals used in the linear mobility law. Some coefficients were found in [27]. Drag parameters for line and climb directions were chosen to be respectively softer and stiffer than the drag parameter of screw dislocations in the prismatic plane.

\begin{tabular}{|l|l|l|}
\hline Drag Coefficient $($ Pa.s $)$ & Drag parameter name & Slip system \\
\hline $1.65 \times 10^{-3}$ & $B_{s}^{\text {glide }}$ & $1 \mathrm{Ba}, 2 \mathrm{Ba}, 3 \mathrm{Ba}$ \\
\hline $7.15 \times 10^{-4}$ & $B_{s}^{\text {glide }}$ & $1 \mathrm{Pr}, 2 \mathrm{Pr}, 3 \mathrm{Pr}$ \\
\hline $1.05 \times 10^{-3}$ & $B_{s}^{\text {glide }}$ & $1 \mathrm{Py}, 2 \mathrm{Py}, 3 \mathrm{Py}$ \\
\hline $2.37 \times 10^{-2}$ & $B_{s}^{\text {glide }}$ & $10 \mathrm{Pr}$ \\
\hline $2.37 \times 10^{-2}$ & $B_{s}^{\text {glide }}$ & $4 \mathrm{Pr}, 5 \mathrm{Pr}, 6 \mathrm{Pr}, 7 \mathrm{Pr}, 8 \mathrm{Pr}, 9 \mathrm{Pr}$ \\
\hline $2.37 \times 10^{-2}$ & $B_{s}^{\text {glide }}$ & $4 \mathrm{Py}, 5 \mathrm{Py}, 6 \mathrm{Py}, 7 \mathrm{Py}, 8 \mathrm{Py}, 9 \mathrm{Py}$ \\
\hline $2.37 \times 10^{\text {climb }}=B_{e}^{\text {climb }}=B^{\text {sessile }}$ & sessile plane \\
\hline $1.18 \times 10^{-3}$ & $B_{s}^{\text {glide }}$ & $1 \mathrm{Ba}, 2 \mathrm{Ba}, 3 \mathrm{Ba}$ \\
\hline $5.70 \times 10^{-4}$ & $B_{e}^{\text {glide }}$ & $1 \mathrm{Pr}, 2 \mathrm{Pr}, 3 \mathrm{Pr}$ \\
\hline $7.28 \times 10^{-4}$ & $B_{e}^{\text {glide }}$ & $1 \mathrm{Py}, 2 \mathrm{Py}, 3 \mathrm{Py}$ \\
\hline $2.37 \times 10^{-2}$ & $B_{e}^{\text {glide }}$ & $10 \mathrm{Pr}$ \\
\hline $2.37 \times 10^{-2}$ & $B_{e}^{\text {glide }}$ & $4 \mathrm{Pr}, 5 \mathrm{Pr}, 6 \mathrm{Pr}, 7 \mathrm{Pr}, 8 \mathrm{Pr}, 9 \mathrm{Pr}$ \\
\hline $2.37 \times 10^{-2}$ & $B_{e}^{\text {glide }}$ & $4 \mathrm{Py}, 5 \mathrm{Py}, 6 \mathrm{Py}, 7 \mathrm{Py}, 8 \mathrm{Py}, 9 \mathrm{Py}$ \\
\hline $2.37 \times 10^{\text {glide }}$ & $B_{e}^{\text {gle }}$ & line direction \\
\hline $5.00 \times 10^{-5}$ & $B^{\text {line }}=B^{\text {jct line }}$ & \\
\hline & & \\
\hline
\end{tabular}

\subsection{Formation and stability of composite dislocations}

Adding and subtracting any two Burgers vectors $\boldsymbol{b}_{A}$ and $\boldsymbol{b}_{B}$ listed in Tables 1- 3 supplies an exhaustive list of possible products of binary dislocation reactions $\boldsymbol{b}_{C}=\boldsymbol{b}_{A} \pm \boldsymbol{b}_{B}$. If formed in a collision between two dislocations gliding in slip planes with unit normals $\boldsymbol{n}_{A}$ and $\boldsymbol{n}_{B}$, the line direction and the glide plane of the product dislocation are $\boldsymbol{t}_{C}=\frac{\boldsymbol{n}_{A} \times \boldsymbol{n}_{B}}{\left|\boldsymbol{n}_{A} \times \boldsymbol{n}_{B}\right|}$ and $\boldsymbol{n}_{\boldsymbol{C}}=\frac{\boldsymbol{t}_{C} \times \boldsymbol{b}_{C}}{\left|\boldsymbol{t}_{C} \times \boldsymbol{b}_{C}\right|}$, respectively.

As has been repeatedly demonstrated in earlier DD simulation studies [3,28], dislocations move, collide and react naturally under the combined action of external stress and dislocation interaction forces. However, in HCP materials, some of the energetically favorable dislocation reactions lead to the formation 
of composite dislocations with Burgers vectors larger than the unit vectors of the HCP lattice. Even $<\mathrm{c}+\mathrm{a}>$ dislocations can themselves be viewed as composites of primary $<\mathrm{a}>$ and $\langle\mathrm{c}>$ dislocations. Physically consistent simulations of composite dislocations require additional algorithmic elements not present to date in DD simulations of cubic crystals.

Qualitatively, the propensity of a given pair of dislocations to form a stable reaction product is often evaluated from Frank's criterion comparing the sum of the energies of the reactant dislocations to the energy of the resulting product. Given that the elastic self-energy of a dislocation is proportional to the square of its Burgers vector, a reaction is assumed to reduce the elastic energy if $\boldsymbol{b}_{A}^{2}+\boldsymbol{b}_{B}^{2}>\left(\boldsymbol{b}_{A}+\boldsymbol{b}_{B}\right)^{2}$. Thus, a pair of dislocations such that $\boldsymbol{b}_{A} \cdot \boldsymbol{b}_{B}<0$ is predicted to attract each other. Similarly when $\boldsymbol{b}_{A} \cdot \boldsymbol{b}_{B}>0$, the dislocation pair is expected to repel. Frank's criterion is quantitatively accurate only in the highly idealized special case of two infinite straight dislocation lines interacting in an isotropic elastic medium with a Poisson's ratio $\nu=0$. However even with these caveats; $\boldsymbol{b}_{\langle c\rangle} \cdot \boldsymbol{b}_{<a\rangle}=0$ thus the stability of a composite $<\mathrm{c}+\mathrm{a}>$ dislocation is uncertain and depends on the actual spatial orientations (characters) of two dislocation lines.

To further examine conditions governing the energetic stability of $<\mathrm{c}+\mathrm{a}\rangle$ dislocations, we rely on Kroupa's formula [29] for the interaction force acting between two infinite straight dislocations in an elastically isotropic solid with the same line direction $\boldsymbol{t}$ and with Burgers vectors $\boldsymbol{b}_{c}=\langle c\rangle$ and $\boldsymbol{b}_{a}=\langle a\rangle$ respectively $[30,29]$ :

$$
\begin{aligned}
\frac{F_{R}}{L} & =\frac{\mu}{2 \pi R}\left[\left(\boldsymbol{b}_{c} \cdot \boldsymbol{t}\right)\left(\boldsymbol{b}_{a} \cdot \boldsymbol{t}\right)+\frac{1}{1-\nu}\left(\boldsymbol{b}_{c} \times \boldsymbol{t}\right)\left(\boldsymbol{b}_{a} \times \boldsymbol{t}\right)\right] \\
\frac{F_{\theta}}{L} & =\frac{\mu}{2 \pi(1-\nu) R^{3}}\left\{\left(\boldsymbol{b}_{c} \cdot \boldsymbol{R}\right)\left[\left(\boldsymbol{b}_{a} \times \boldsymbol{R}\right) \cdot \boldsymbol{t}\right]+\left(\boldsymbol{b}_{a} \cdot \boldsymbol{R}\right)\left[\left(\boldsymbol{b}_{c} \times \boldsymbol{R}\right) \cdot \boldsymbol{t}\right]\right\}
\end{aligned}
$$

where $\boldsymbol{R}$ is the shortest vector connecting two dislocation lines, i.e. $\boldsymbol{R} \cdot \boldsymbol{t}=0$, $R=|\boldsymbol{R}|, F_{R}$ is the radial component of the interaction force, and $F_{\theta}$ is the angular component (torque).

As immediately follows from the above equations, the radial component of the interaction force $F_{R}$ depends only on the magnitude but not on the orientation of vector $\boldsymbol{R}$ (note that the torque force does depend on the orientation of vector $\boldsymbol{R}$ with respect to $\boldsymbol{c}$ and to $\boldsymbol{a}$ ). Fig. 2 is a contour plot of $F_{R}$ as a function of line direction vector $\boldsymbol{t}$ defined by spherical angles $\theta$ and $\phi$ counted from a polar axis parallel to $\boldsymbol{c}$ and from an azimuthal reference direction parallel to $\boldsymbol{a}$, respectively. As seen from Fig. 2, the radial interaction force is positive (repulsive) for exactly half of possible line orientations $\boldsymbol{t}$ indicating that the composite $<\mathrm{c}+\mathrm{a}>$ dislocation is elastically unstable for those line orientations. For the other half of line orientations, the interaction force is negative 
(attractive), and the composite $<\mathrm{c}+\mathrm{a}>$ dislocation is elastically stable.

Although this analysis of the stability of $\langle\mathrm{c}+\mathrm{a}\rangle$ dislocations is conducted in an elastically isotropic medium, similar analyses have been conducted for $<\mathrm{c}+\mathrm{a}>$ dislocations in anistropic media $[10,16]$. Although Yoo et. al [16] limited their analysis to a single crystallographic plane, they similarly found directions at which the $<\mathrm{c}+\mathrm{a}>$ dislocation was elastically unstable in anistropic media. Furthermore, their analysis revealed that the directions at which the $<\mathrm{c}+\mathrm{a}>$ dislocation transitioned from elastically stable to unstable did not change as a function of elastic anisotropy, but that the strength of the repulsion and attraction did. Likewise, in our results we would expect that the strength of the repulsion and attraction would change with the use of anisotropic elasticity, but the observation that the $\langle\mathrm{c}+\mathrm{a}\rangle$ dislocation is elastically unstable for half of the line orientations on a sphere would remain.

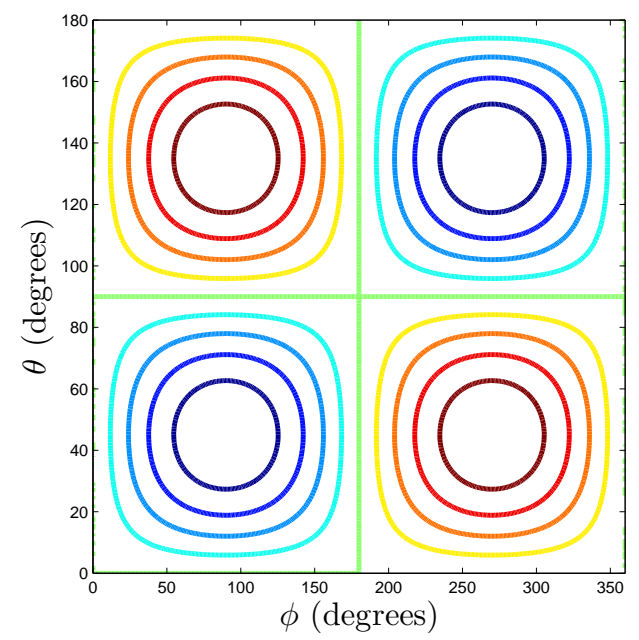

Fig. 2. Contour plot of the radial component of the interaction force between two infinite straight dislocations of $<\mathrm{a}>$ and $<\mathrm{c}>$ types in $\mathrm{HCP}$ beryllium as a function of angles $\theta$ and $\phi$ defining the spherical coordinates of line direction $\boldsymbol{t}$.

In addition to the elastic interaction between a pair of dislocations, the energetic balance of dislocation reactions can depend on external stress and on details of the atomic arrangement in the dislocation cores. For instance, it may be the case that the core energy of the $\langle\mathrm{c}+\mathrm{a}\rangle$ product dislocation is lower than the sum of the core energies of $\langle a\rangle$ and $\langle\mathrm{c}\rangle$ dislocations. In such $a$ case, the balance of core energies may hold the product dislocation together even when the elastic force (Eq. 14) between the two component dislocations is repulsive. In the absence of necessary atomistic data, one must rely on the elastic interaction alone in deciding whether a given composite dislocation is energetically stable or unstable.

In addition to the direction and magnitude of the elastic interaction force 
between a pair of dislocations, detailed outcomes of dislocation reactions in 3D depend on the glide planes, incidence angles of two colliding lines, external stress, and availability of planes of easy glide of the interacting dislocations. In particular, the results of binary reactions that form $<\mathrm{c}+\mathrm{a}\rangle$ dislocations are believed to involve collisions of $\langle\mathrm{a}\rangle$ and $\langle\mathrm{c}\rangle$ dislocations in their shared prismatic plane followed by cross-slip of the $<\mathrm{c}+\mathrm{a}\rangle$ composite dislocation into a pyramidal plane [16]. Formation of $\langle\mathrm{c}+\mathrm{a}\rangle$ dislocations is believed to be additionally enhanced near external surfaces and internal interfaces such as grain boundaries and incoherent twin boundaries [16].

$<\mathrm{c}+\mathrm{a}>$ dislocations are just one type of composite dislocation that may be encountered in DD simulations of HCP materials. Composite dislocations may also result from energetically-favorable binary reactions between $<\mathrm{c}+\mathrm{a}\rangle$ dislocations in HCP crystals. There are eight distinct types of binary reactions between two $\langle\mathrm{c}+\mathrm{a}\rangle$ dislocations taking into account the symmetry of the HCP lattice. Of them, only four are elastically attractive, and of the four, two reactions lead to the formation of other composite dislocations. Representative examples of the four attractive $\langle\mathrm{c}+\mathrm{a}\rangle$ binary reactions are listed in Table 5 . The attraction of the two parent dislocations in Table 5 is robust in the sense that both the Frank's rule and the Kroupa's formula predict attractive interaction between two parent dislocations irrespective of the line orientation. For such reactions, Frank's criterion is sufficient to discriminate among attractive and repulsive reactions. The first two reactions in the table lead to a complete annihilation and an $<\mathrm{a}>$ type product dislocation, respectively. The last two reactions in the table lead to the formation of intermediate composite product dislocations. The third reaction leads to $\mathrm{a}<\mathrm{d}>$ type dislocation with a $<\overline{1} 010>$ Burgers vector, and the fourth reaction leads to a $<2 \mathrm{a}>$ dislocation. Again, according to Frank's criterion and Kroupa's formula, both of these composite product dislocations are elastically unstable for all line directions when compared to the final products listed in the fourth collumn of Table 5. Thus, when they appear in DD simulations of HCP crystals they should be immediately dissociated unless there are mobility constraints that would prevent their dissociation.

\subsection{Topological operators for dislocation dissociation}

When the interaction force between component dislocations that make up the composite dislocation is repulsive, the DD model should allow the splitting of the composite dislocation into its components. This need is largely specific to $\mathrm{HCP}$ and to possibly other low-symmetry crystals and does not normally arise in DD simulations of high-symmetry cubic materials where dislocation collisions should not lead to the formation of composite dislocations. The ParaDiS code relies on two elementary topological operators - splitnode and mergenode 
Table 5

Reactions Burgers vectors that result from attractive pairs of $\langle\mathrm{c}+\mathrm{a}\rangle$ type dislocations. In the list below, the intermediate product Burgers vectors are energetically favorable with respect to the initial parents and the final products Burgers vectors are energetically favorable with respect to the intermediate products.

\begin{tabular}{|c|c|c|c|}
\hline Burgers vector 1 & Burgers vector 2 & Intermediate product & Final product \\
\hline $1 / 3[\overline{1} \overline{1} 23]$ & $1 / 3[1 \overline{1} \overline{2} \overline{3}]$ & 0 & 0 \\
\hline $1 / 3[\overline{1} \overline{1} 23]$ & $1 / 3[2 \overline{1} \overline{1} \overline{3}]$ & $1 / 3[1 \overline{2} 10]$ & $1 / 3[1 \overline{2} 10]$ \\
\hline $1 / 3[\overline{1} \overline{1} 23]$ & $1 / 3[\overline{2} 11 \overline{3}]$ & {$[\overline{1} 010]$} & $1 / 3[\overline{1} \overline{1} 20]+1 / 3[\overline{2} 110]$ \\
\hline $1 / 3[\overline{1} \overline{1} 23]$ & $1 / 3[\overline{1} \overline{1} 2 \overline{3}]$ & $2 / 3[\overline{1} \overline{1} 20]$ & $1 / 3[\overline{1} \overline{1} 20]+1 / 3[\overline{1} \overline{1} 20]$ \\
\hline
\end{tabular}

- to add or remove nodal degrees of freedom and to change the connectivity of an evolving dislocation network as required for handling dislocation core reactions $[3,21]$. The mergenode operator combines two nodes into one while the splitnode operator creates a new node and moves some of the connections from an existing node to the new one. Since the splitnode operator has been defined so that the Burgers vectors of the existing arms do not change during the operation, an additional topological operator splintersegment is needed to change the Burgers vector of a connection and to aid the splitnode operator in handling the dissociation of composite dislocations into two component dislocations when appropriate.

An example of the splinter segment operator in action is given in Figs. 3[a]-[c]. When invoked, splintersegment replaces the segment connecting two neighboring nodes $\boldsymbol{p}$ and $\boldsymbol{q}$ (Fig. 3[a]) with two segments creating a double connection between the nodes. The same operator partitions the composite Burgers vector of the original segment into two component Burgers vectors, assigns them to the two new segments and assigns glide planes to the new segments. splintersegment is then invoked again to perform a similar sequence on the neighboring segment connecting nodes $\mathbf{0}$ and $\boldsymbol{p}$ resulting in the intermediate configuration found in Fig. $3[\mathrm{~b}]$. Now that node $\boldsymbol{p}$ has four connections, the splitnode operator is invoked to split it into nodes $\boldsymbol{p}_{1}$ and $\boldsymbol{p}_{2}$ shown in Fig. 3[c]. The positions of nodes $\boldsymbol{p}_{1}$ and $\boldsymbol{p}_{2}$ must be chosen such that they are placed outside of the annihilation distance used to determine when dislocations are colliding and such that they do not violate any of the mobility restrictions of the lines connected to them.

The splinter segment operator may also aid in the dissociation of a composite dislocation when the composite dislocation is connected to a physical node, connecting three or more segments as depicted in Fig. $3[\mathrm{c}]$. In this example, the splintersegment operation is used to partition the $\boldsymbol{b}$ burgers vector of the segment connecting nodes $\boldsymbol{q}$ and $\mathbf{1}$ into two segments with the burgers vectors $\boldsymbol{b}_{1}$ and $\boldsymbol{b}_{2}$ as shown in the intermediate configuration found in Fig. 3[d]. Again, the splitnode operator is invoked to split node $\boldsymbol{q}$ into nodes $\boldsymbol{q}_{1}$ and $\boldsymbol{q}_{2}$ shown 
in Fig.3[e], and the positions of nodes $\boldsymbol{q}_{1}$ and $\boldsymbol{q}_{2}$ must be chosen such that they are placed outside of the annihilation distance used to determine when dislocations are colliding and such that they do not violate any of the mobility restrictions of the lines connected to them.

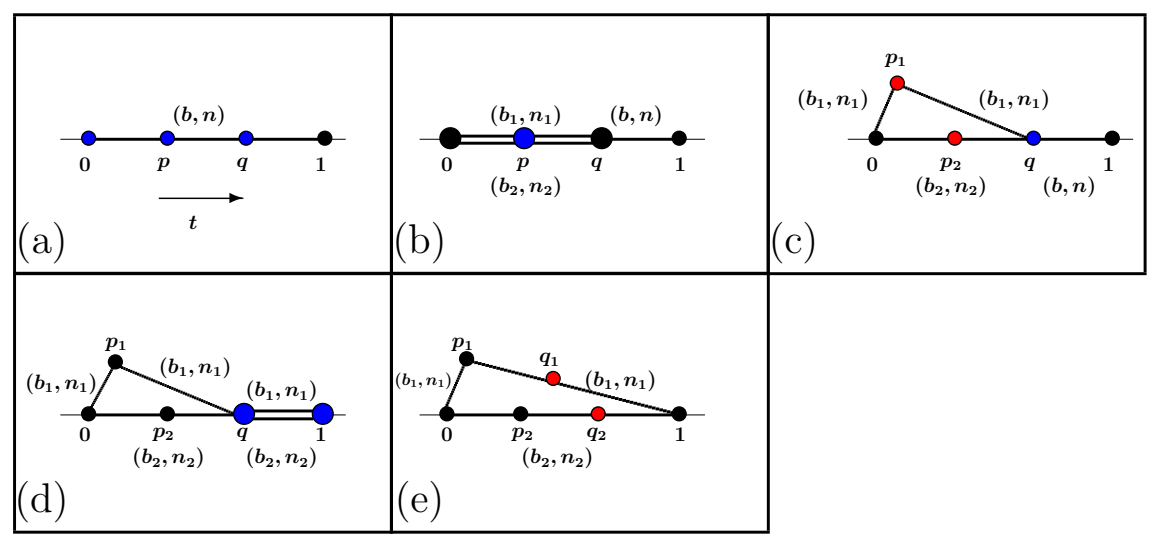

Fig. 3. The sequence of topological operations conducted during a $<\mathrm{c}+\mathrm{a}>$-dissociation into $<\mathrm{c}\rangle$ and $<\mathrm{a}\rangle$ dislocations. (a) Initial topology. Several dislocation segments of type $<\mathrm{c}+\mathrm{a}>$ aligned along $\boldsymbol{t}$. (b) Splintersegment of segments $[\mathbf{0}, \boldsymbol{p}]$ and $[\boldsymbol{p}, \boldsymbol{q}]$. (c) Splitnode operation of node $\boldsymbol{p}$ into $\boldsymbol{p}_{\mathbf{1}}$ and $\boldsymbol{p}_{2}$.(d) Unzipping of node $\boldsymbol{q}$ starts with a splintersegment of segment $[\boldsymbol{q}, \mathbf{1}]$ and a (e) splitnode of node $\boldsymbol{q}$ into two nodes $\boldsymbol{q}_{\mathbf{1}}$ and $\boldsymbol{q}_{\mathbf{2}}$.

\subsection{Criteria for dislocation cross-slip and dissociation}

The splintersegment and splitnode operators modify connections of dislocation nodes, but the decision on when and which nodes to split and what to do with the new nodes after they are created, must rely on separate algorithms. In this section we describe two closely related algorithms: one used to decide when a dislocation changes its glide plane (cross-slips) and another one used to decide when and how to split a composite dislocation.

Under typical straining conditions, dislocation segments are confined to move in their geometric planes of easy glide, i.e. the planes with normal $\boldsymbol{n}_{g}=\boldsymbol{b} \times \boldsymbol{t}$ defined by each segment's line direction $\boldsymbol{t}$ and Burgers vector $\boldsymbol{b}$. Segments in the screw orientation for which $\boldsymbol{t} \| \boldsymbol{b}$ are special since their glide planes are undefined meaning that such segments can in principle glide in any plane containing its Burgers vector. However, for a wide class of materials even screw segments are often confined to well defined glide planes due to their core structure; and although infrequent, transitions from one glide plane to another - cross-slip events - are possible. 
In the HCP model presented in this paper, each dislocation segment - screw or otherwise - is confined to glide only in one of several predefined planes listed in Tables 1- 3. Under the right conditions, the screw segments may prefer to switch from one glide plane to another in the permitted set to reduce the stored elastic energy of the system. Our HCP model prescribes that, out of several permitted glide planes, a screw dislocation moves in the plane where it dissipates the stored elastic energy at the fastest rate, i.e. in the plane in which the product of the projection of the Peach-Koehler force and the segment's velocity is at its maximum.

ParaDiS is a nodal model in the sense that the entire dislocation configuration is represented by nodes connected to other nodes, and it is the motion of the nodes that the algorithm is tracking during a simulation. At the same time, dislocation mobility, including glide plane assignment, is a physical property of dislocation segments. For a discretization node connecting two segments in a primary slip plane to move into a cross-slip plane we require two conditions to be met: (1) both segments should be screw, i.e. aligned (within tolerance) with the Burgers vector $\boldsymbol{b}$ and (2) motion of the node into the cross-slip plane results in a higher dissipation rate than its motion in the current glide plane. As long as the current glide plane of a screw segment enables the highest rate of plastic dissipation, the planes of the connected segments remain unchanged. But if a different plane of the permitted set is found to maximize the dissipation rate, the glide plane assignment for the screw segments changes, and the node glides into the new plane. Thus, for every node deemed to be a "screw node" (condition 1 above), the dissipation rate for every potential glide plane is evaluated, and the glide plane defined in the mobility function of the screw segment is adjusted to enable the screw dislocation to move in the plane which maximizes its plastic dissipation rate. This change in glide plane occurs between time increments and not during the time increment so that stability of the time update procedure is not affected.

Dissociation of composite dislocations is handled in a manner similar to the cross-slip operation. If the current glide plane of the composite dislocation is shared by its two potential dissociation products, dissociation is allowed to occur regardless of the line direction of the composite dislocation in the common plane, whenever motion of component dislocations results in a higher dissipation rate than motion of the (undissociated) composite dislocation. Such is the case for $\mathrm{a}<\mathrm{c}+\mathrm{a}>$ composite dislocation in the common prismatic plane, $\mathrm{a}<2 \mathrm{a}>$ in any of the four listed glide planes for $<\mathrm{a}>$ dislocations, or $\mathrm{a}<\mathrm{d}>$ composite dislocation in the basal plane. However, more complex dissociations where one or both dissociation products move into glide planes that are different from the glide plane of the parent composite dislocation may also occur.

For a node on a composite dislocation to be considered for such a non-planar 
dissociation, the two segments connected to the node should be aligned with at least one of the permitted glide planes of the two component dislocations. Along with the geometric constraints, a set of dynamic criteria must also be met for the dissociation of the composite dislocation to take place. First, similar to the cross slip criterion, the dissociation can be accepted only if the sum of dissipation rates of two dissociation products in their putative glide planes is greater than the dissipation rate of continued motion of the composite dislocation in its current plane. Second, an embryonic configuration of the kind depicted in Fig. 3[c], with one of the resultant new nodes displaced by the distance slightly greater than the annihilation distance in a direction perpendicular to the original line and contained within the glide plane of the component dislocation, must sweep a positive area. In other words, the time rate of change in area defined by nodes $\mathbf{0}, \boldsymbol{p}_{1}$, and $\boldsymbol{q}$ in Fig. 3[c] must be positive. When both criteria are met the splintersegment and splitnode operators are invoked. The node is logically split, and the embryonic configuration that was used in the evaluation of the criteria is created. In cases when more than one dissociation mode meets the criteria, the mode with the greatest dissipation rate is accepted. Examples of dissociation events conforming to these criteria are presented in section 3.1.

We note that composite dislocations have been observed to occasionally form even in DD simulations of high-symmetry cubic crystals where their formation is not favored energetically. Their occurrence may result from large local stresses generated by groups of dislocations, forcing some of them to merge against their mutual repulsion. Whatever the cause, once formed such composite dislocations persist though DD simulations lacking a mechanism for their dissociation. Thus, enabling dissociation mechanisms of the kind discussed here should generally improve fidelity of DD simulations for all types of crystal lattices.

\section{Simulation results and discussion}

The following sections present results of dislocation dynamics simulations intended to illustrate the formation, dissociation and strength of composite product dislocations in HCP beryllium and the collective behavior of large ensembles of dislocations subjected to a constant rate straining. In addition to the dislocation mobility and topological operations defined in the preceding sections, the DD simulations employ isotropic elasticity. A shear modulus of $\mu=144 \mathrm{MPa}$, a Poisson's ratio of $\nu \approx 0.064$ were used. Beryllium is considered to be a mildly anisotropic elastic crystal with an anistropy ratio of 1.2 using different measures [31,32], and the isotropic approximation led to a significant savings in computational expense of the simulations. A $c / a$ ratio of 1.568 , and a lattice constant of $a=2.29 \dot{A}$ consistent with room temperature beryllium 
were used.

\subsection{Dissociation of composite dislocations of $\langle c+a\rangle$-type}

DD simulations are conducted to test the dissociation of a Frank-Read source of $\mathrm{a}<\mathrm{c}+\mathrm{a}>$ type dislocation into two primary $\langle\mathrm{c}\rangle$ and $\langle\mathrm{a}\rangle$ dislocations. These Frank-Read source examples are specifically chosen to be of particular mixed character $<\mathrm{c}+\mathrm{a}>$ dislocations to illustrate the dissociation of composite dislocations into cross-slip planes. The stress conditions applied are specifically chosen to also promote their dissociation.

The first DD simulation consists initially of a Frank-Read source dislocation in the slip system $8 \mathrm{Pr}$, i.e. of Burgers vector type $<\mathrm{c}+\mathrm{a}>$ with the line direction $\boldsymbol{t}$ along $\boldsymbol{b}_{10}$.

Stress of the form

$$
\boldsymbol{\sigma}=\alpha\left[\frac{\mathbf{S}_{\mathbf{A}}-\left(\mathbf{S}_{\mathbf{A}}: \mathbf{S}_{\mathbf{B}}\right) \mathbf{S}_{\mathbf{B}}}{1-\left(\mathbf{S}_{\mathbf{A}}: \mathbf{S}_{\mathbf{B}}\right)^{2}}\right]
$$

is applied to the Frank-Read source. In Eq. 16,

$$
\boldsymbol{S}_{i}=\frac{1}{\sqrt{2}}\left(\frac{\boldsymbol{b}_{i}}{\left|\boldsymbol{b}_{i}\right|} \otimes \frac{\boldsymbol{n}_{i}}{\left|\boldsymbol{n}_{i}\right|}+\frac{\boldsymbol{b}_{i}}{\left|\boldsymbol{b}_{i}\right|} \otimes \frac{\boldsymbol{n}_{i}}{\left|\boldsymbol{n}_{i}\right|}\right), i=A, B
$$

where $\left(\boldsymbol{b}_{A}=\boldsymbol{b}_{10}, \boldsymbol{n}_{A}=(01 \overline{1} 0)\right)$ and $\left(\boldsymbol{b}_{B}=\boldsymbol{b}_{8}, \boldsymbol{n}_{B}=(\overline{1010)})\right.$. This applied stress is such that $\alpha$ is the amount of stress needed to bow the dislocation out. It is defined such that the $8 \mathrm{Pr}$ slip system sees a zero resolved shear stress, and such that the $\langle\mathrm{a}\rangle$ and $\langle\mathrm{c}\rangle$ have non-zero resolved shear stress on their glide planes.

Fig. $4[\mathrm{a}-\mathrm{b}]$ shows the initial composite $<\mathrm{c}+\mathrm{a}>$-type dislocation dissociating into primary dislocations of type $<\mathrm{a}>$ and $\langle\mathrm{c}>$, respectively. Each dislocation component glides on a different plane. The dislocation of type $<\mathrm{c}>$ continues to glide on the same prismatic plane as the initial $<\mathrm{c}+\mathrm{a}>$ dislocation, 10PrI. The dislocation of type $<\mathrm{a}>$ now belongs to the $3 \mathrm{Pr}$ slip system and glides away from the source on that prismatic plane.

The second DD simulation consists of a Frank-Read source dislocation in the slip system 8PyII with line direction $\boldsymbol{t}$ along [1101]. The applied stress defined in this case is of the form given in Eq. 16 with the slip systems $\left(\boldsymbol{b}_{A}=\boldsymbol{b}_{3}\right.$, $\left.\boldsymbol{n}_{A}=(10 \overline{1} 1)\right)$ and $\left(\boldsymbol{b}_{B}=\boldsymbol{b}_{8}, \boldsymbol{n}_{B}=(0 \overline{1} 11)\right)$. Under this applied stress, the composite $<\mathrm{c}+\mathrm{a}>$-type dislocation dissociates into an $<\mathrm{a}\rangle$ dislocation in the 3 PyI slip system and a $<\mathrm{c}>$ dislocation on the $(11 \overline{2} 0)$ prism plane. The $(11 \overline{2} 0)$ prismatic plane is not a glide plane in our HCP model, so the $<\mathrm{c}>$ dislocation 
is unable to glide away from the initial source position. The $<a>$ dislocation is glissile and bows out.

The $<\mathrm{c}+\mathrm{a}>$-dissociation procedure results in configurations that cannot be observed in other crystals and is particular to HCP materials. In the first simulation, a Frank-Read source ends up bowing out two dislocations into two different glide planes. This corresponds to dislocation partially cross slipping into two different glide planes. In the second simulation, one of the two component dislocations produced through dissociation of a non-screw $<\mathrm{c}+\mathrm{a}>$ composite dislocation, bows out on its glide plane leaving behind another sessile component dislocation. It is unclear how often such mechanisms may be invoked during the bulk deformation of HCP metals, but their crystalline geometry permits them to occur. A dislocation dynamics code should allow such topological operations to occur if they act to relax the stored elastic energy of the dislocation microstructure.

\subsection{Formation and dissociation of composite product dislocations in $3 D$}

The DD model can be used to study the collisions of two attractive $<\mathrm{c}+\mathrm{a}>$ dislocations resulting in the formation of $<2 \mathrm{a}>$ junction product for three different combinations of glide planes of the parent dislocations. This illustrates the intermediate and final reactions in the last line of Table 5.

Consider two parent dislocations with Burgers vectors $\boldsymbol{b}_{6}=1 / 3[\overline{1} \overline{1} 23]$ and $\boldsymbol{b}_{7}=1 / 3[\overline{1} \overline{1} 2 \overline{3}]$. The Burgers vector of a product dislocation formed in their reaction is $\boldsymbol{b}_{\text {product }}=\frac{2}{3}[\overline{1} \overline{1} 20]$. According to Frank's rule, such a reaction leads to a reduction in the squared Burgers vector $\left|\boldsymbol{b}_{6}\right|^{2}+\left|\boldsymbol{b}_{7}\right|^{2}=2\left(a^{2}+c^{2}\right)>|\boldsymbol{b}|^{2}=$ $4 a^{2}$, for $c>a$, and is energetically favored. At the same time, the Burgers vector of the product dislocation $\boldsymbol{b}_{\text {product }}$ is only an intermediate product and is twice the Burgers vector of a primary $<\mathrm{a}\rangle$ dislocation and should further dissociate into two $<\mathrm{a}>$ dislocations. In the notation defined in Tables 1- 3, the expected total dislocation reaction can be written as

$$
\boldsymbol{b}_{6}+\boldsymbol{b}_{7} \rightarrow \boldsymbol{b}_{2}+\boldsymbol{b}_{2}
$$

In Fig. 5[a], we consider the collision of two $<\mathrm{c}+\mathrm{a}>$ dislocations with Burgers vectors $\boldsymbol{b}_{6}$ and $\boldsymbol{b}_{7}$ on two different pyramidal planes, 6PyII and 7PyI, respectively. Both parent dislocations have a $40^{\circ}$-mixed character with respect to the junction axis in their initial orientations. The incidence angle between two intersecting dislocations is sufficiently small leading them to form a $<2 \mathrm{a}>$ junction product in the manner shown in Fig. $5[\mathrm{~b}]$. The resulting junction dislocation resides in the basal (0001) plane and looks exactly like any other junction dislocation previously studied in high-symmetry cubic crystals. How- 

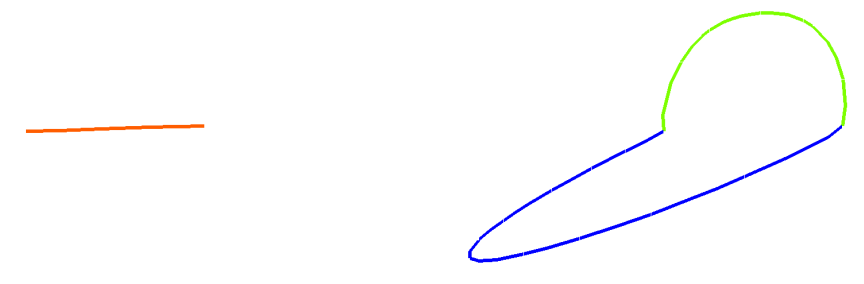

(a) Initial configuration $\boldsymbol{t}=[0001]$

(b) Final configuration
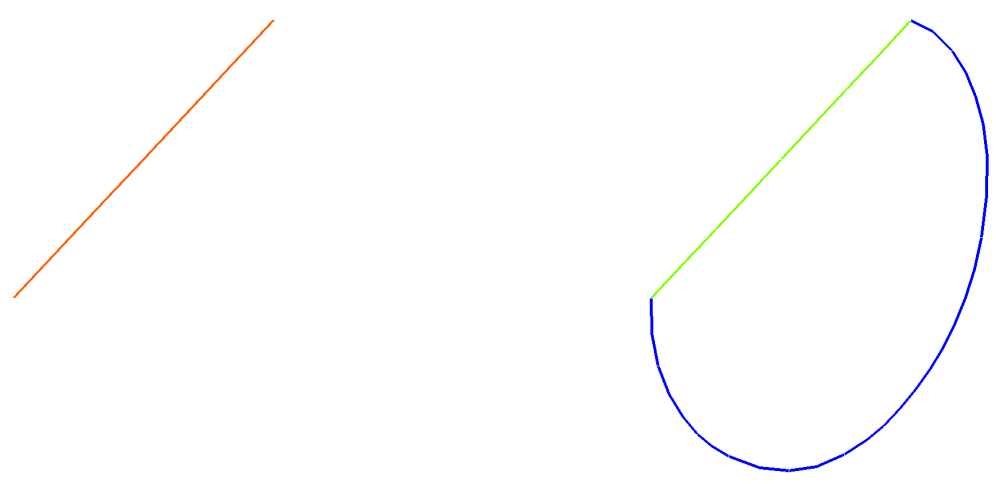

(c) Initial configuration $\boldsymbol{t}=B E^{\prime} /\left|B E^{\prime}\right|$

(d) Final configuration

Fig. 4. Frank-Read source dislocation of Burgers vector type $<c+a>$. Under an applied stress, the dislocation dissociates into two dislocations of type $<\mathrm{a}>$ and $<\mathrm{c}>$. (a-b) Both dislocations $\langle\mathrm{c}\rangle$ and $<\mathrm{a}>$ are mobile and bow out in their respective glide planes. (c-d) The dislocation of type $<c>$ is sessile and remains in the original position of the $<\mathrm{c}+\mathrm{a}>$ dislocation. The dislocation of type $<\mathrm{a}>$ is mobile and bows out. Orange is a dislocation of type $\langle\mathrm{c}+\mathrm{a}\rangle$, blue and green are dislocations of type $<\mathrm{a}>$ and $<\mathrm{c}>$ respectively.

ever, in our DD model of HCP crystals, this composite junction dislocation is able to dissociate into two $<\mathrm{a}>$ dislocations on the basal plane. The end result is the configuration shown in Fig. $5[\mathrm{c}]$ where two $<\mathrm{a}>$ dislocations completely replace the original $<2 \mathrm{a}>$ junction leading to a considerable reduction in the elastic energy of the configuration. The 4-nodes at the junction's ends are stable against further dissociation into 3-nodes. The stability of this 4-node is similar to the stability of a 4-node formed by two intersecting dislocations for a range of angles of intersection.

In a second simulation, the first dislocation is placed in the same pyramidal plane as before, 6PyII, and the second dislocation is placed in its prismatic 
plane, 7Pr, as shown in Fig. 5[d]. The resulting $<2 \mathrm{a}>$ junction dislocation is contained in the same prismatic plane as one of the parents as shown in Fig. 5[e]. As the $<2 \mathrm{a}>$ dislocation dissociates into two $<\mathrm{a}>$ primary dislocations, both 4-nodes bounding the junction split further into 3-nodes connected by $<\mathrm{c}>$ dislocation segments as shown in Fig. 5[f]. Since three of the four dislocations entering the 4-node share a glide plane, a lower energy configuration can be reached by further dissociating of the 4-node into two 3-nodes and extending a secondary $<\mathrm{c}>$ junction in the same prismatic plane.

In the third simulation, both parent dislocations are initially placed in the same prismatic plane, $6 \operatorname{Pr}$ and $7 \operatorname{Pr}$ respectively, so that the product dislocation finds itself in the same prismatic plane as its parents, as shown in Fig. 5 [g]. The end result of this simulation is shown in Fig. 5[i]. Although the two 4-nodes are now glissile in the prismatic plane, their dissociation into two 3-nodes connected by $<\mathrm{c}>$ dislocations is again found to reduce the energy proceeding in a manner similar to the second case:

$$
\begin{aligned}
& \boldsymbol{b}_{6} \rightarrow \boldsymbol{b}_{2}+\boldsymbol{b}_{10} \\
& \boldsymbol{b}_{7} \rightarrow \boldsymbol{b}_{2}-\boldsymbol{b}_{10} .
\end{aligned}
$$

For comparison, Fig. 5[h] shows the end result of the same reaction but with the dissociation disallowed.

In all three pairwise reactions between $\langle\mathrm{c}+\mathrm{a}\rangle$ dislocations considered here, the product $<2 \mathrm{a}>$ dislocation splits into two $<\mathrm{a}>$ dislocations. In the first case, the 4-nodes at the junction's ends remain together due to mobility constraints. In the other two cases, our DD simulation naturally dissociates the 4nodes into 3-nodes and extends a secondary $<\mathrm{c}>$ junction dislocation resulting in a more complex and more elastically stable final dislocation configuration as a result of the binary collision.

\subsection{Strength of composite junctions}

DD simulations were conducted to calculate the critical stress required to unzip a junction formed by a collision of two $<c+a>$ dislocations coming into contact by glide in two different planes and forming a $<2 \mathrm{a}>$ product junction dislocation versus two $<\mathrm{a}>$ product junction dislocations.

The strength of a dislocation junction can be described in terms of its yield surface, a five-dimensional surface in the six-dimensional space spanned by the components of stress applied on the junction. A concept borrowed from the field of continuum crystal plasticity, a yield surface is a convex hull with each of 


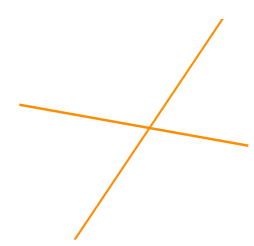

(a) Initial configuration

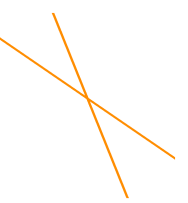

(d) Initial configuration

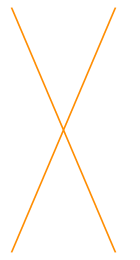

(g) Initial configuration

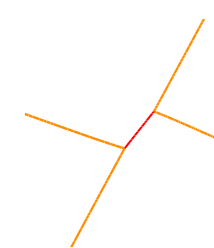

(b) No dissociation

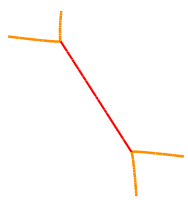

(e) No dissociation

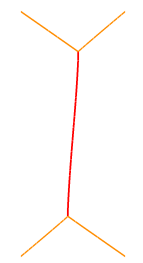

(h) No dissociation

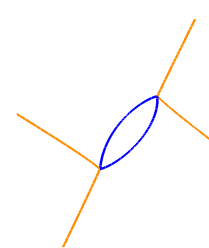

(c) Dissociation allowed

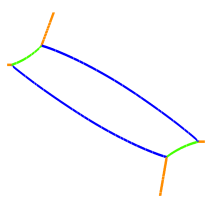

(f) Dissociation allowed

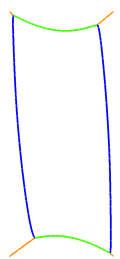

(i) Dissociation allowed

Fig. 5. Dislocation dynamics simulations of the junction formation between two $<\mathrm{c}+\mathrm{a}>$-type dislocations. The interacting dislocations have Burgers vector $\boldsymbol{b}_{6}$ and $\boldsymbol{b}_{7}$. The junction Burgers vector is $2 \boldsymbol{b}_{\mathbf{2}}$ which can be split to two Burgers vectors of type $\boldsymbol{b}_{2}$. The first column corresponds to the initial configurations. The middle column corresponds to the resultant configurations when no composite dislocation dissociation occurs. The last column corresponds to the resultant configuration when dissociation of composite dislocations is allowed. Orange color depicts the $<\mathrm{c}+\mathrm{a}\rangle$ dislocations. Blue and green colors depict the $\langle a\rangle$ and $\langle\mathrm{c}\rangle$ dislocations respectively, and red is used to depict a composite dislocation of type $\langle 2 a\rangle$. The planes differ in each simulations. (a-c) The two initial dislocations are on two different pyramidal planes, the resulting junction is on a basal plane. (d-f) The two initial dislocations are on a pyramidal and a prismatic planes respectively. The resulting junction is on the same prismatic plane as one of the parents. (g-i) All three dislocations are on the same prismatic plane.

its points representing the minimal (or critical) magnitude of stress required to unzip, or yield the junction when stress is applied along the 5-vector connecting the origin to this point. When it comes to binary dislocation junctions, only three Schmid tensor projections of a general stress tensor matter: one for each of two parent dislocations, and one for the product junction dislocation. Thus, it is sufficient and convenient to represent the yield surface of a binary junction in terms of three linearly independent stress projections each of which interacts only with one of the three participating dislocations. (It is possible 
to construct such three independent projections for most, but not all junction geometries. One special geometry where only two projections can be defined is the co-planar junction where all three dislocations share the same glide plane.)

Following the procedure described in [33,34], the critical stress to break a junction is written as

$$
\boldsymbol{\sigma}=\alpha \boldsymbol{S}_{<c+a>}^{1}+\beta \boldsymbol{S}_{<c+a>}^{2}+\gamma \boldsymbol{S}_{<a>}
$$

where parameters $\alpha, \beta$ and $\gamma$ are the magnitudes of stress projected on the Schmid tensors of two parent $\langle\mathrm{c}+\mathrm{a}\rangle$ dislocations and the $<2 \mathrm{a}\rangle$ product dislocation, respectively. Here we only consider the two-dimensional crosssection of the yield surface corresponding to $\gamma=0$.

Shown in Fig. 6 are two yield contours of critical stress $\boldsymbol{\sigma}$ (as defined in Eq. 18) computed for configurations Fig. 5[b] and Fig. 5[c], respectively. Quite clearly, the strength of the junction depicted in Fig. $5[\mathrm{~b}]$, where the product dislocation remains undissociated, is markedly lower than the strength of the dissociated junction on Fig. 5[c]. Averaged over all combinations of $\alpha$ and $\beta$, the strength of the former is only about $2 / 3$ of the strength of the latter. The increased strength of the dissociated junction is due to the lower initial energy of this configuration requiring higher stress to unzip. This comparison suggests that simulations of HCP crystals that do not enable the dissociation of composite dislocations may systematically and unphysically underestimate the strength and strain hardening due to forest interactions of $\langle\mathrm{c}+\mathrm{a}\rangle$ dislocations.

\subsection{Simulations of bulk crystal plasticity}

Dislocation dynamics simulations of single crystal beryllium subjected to three different straining conditions are presented. These simulations verify that the new components implemented in the ParaDiS code specifically for HCP materials (elastic forces, core energies, mobility laws, topological operations, etc.) integrate to produce new insights into the physics for crystal plasticity of HCP crystals and provide output in the form of coarse-grained measures of microstructural evolution valuable in the construction of mesoscopic continuum models.

The dislocation mobility parameters used in the DD simulations are given in Table 4. The loading conditions of three simulations of bulk single crystal beryllium reported here are: (1) simple shear in the basal plane along the

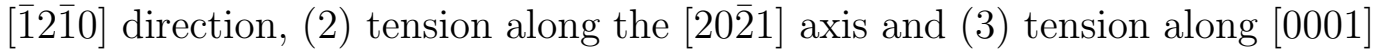
axis. All three simulations were performed at the same straining rate of $10^{5} / \mathrm{s}$. The initial dislocation structure consists of sets of octagonal and hexagonal (for prism planes only) prismatic loops with a constant radius of 5, 000b. These 


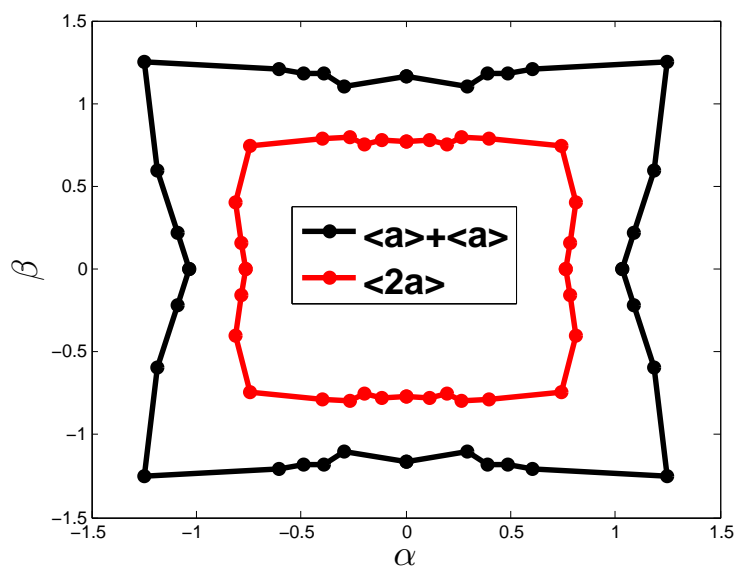

Fig. 6. Yield surface comparison between $\langle 2 a\rangle$ dislocation and $\langle a\rangle+\langle a\rangle$ dislocation junctions. The junctions formed in the case of Fig. $5[\mathrm{~b}]$ and $[\mathrm{c}]$ are used as initial configurations. The axis correspond to the amount of stress $\alpha$ and $\beta$ resolved on the parent $<\mathrm{c}+\mathrm{a}>$ dislocation glide planes needed to break the junction.

loops are randomly distributed in all possible slip systems listed in Tables 1-3, with each slip system having the same initial dislocation density. The initial dislocation density in the $3 \times 3 \times 3 \mu \mathrm{m}^{3}$ computational box (for the cases of basal shear and $<$ a $>$-axis tension) is $4 \times 10^{12} \mathrm{~m}^{-2}$.

For the $<\mathrm{c}>$-axis loading case where the $5 \times 5 \times 5 \mu \mathrm{m}^{3}$ box is used, the initial dislocation density is $9 \times 10^{12} \mathrm{~m}^{-2}$. Periodic boundary conditions are used for dislocations crossing the computational box boundaries.

The stress-strain curves obtained in the three simulations are plotted in Fig. 7[a], and the associated evolution of dislocation densities are shown in Fig. 7[b]. Figs. 8- 12 present the time-evolution of dislocation densities partitioned by Burgers vector and of dislocation flux components resolved by the slip system extracted from these simulations. Appendix B details methods used to partition the total dislocation density and the total dislocation flux over individual Burgers vectors and slip systems.

Little strain hardening is observed in Fig. $7[\mathrm{a}]$ when the system is sheared in the basal plane. The time-evolution of dislocation density components in Fig. 8[a] and slip systems fluxes in Fig. 8[b] reveal that only one slip system is actively responding to shear, namely the most highly stressed 2Ba system, leading to negligible dislocation multiplication under this single slip deformation mode. 


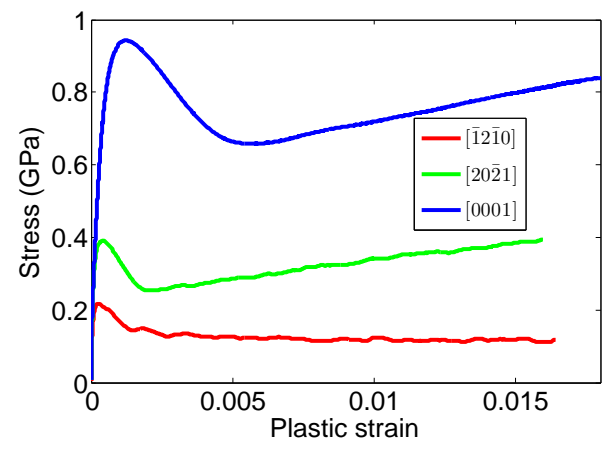

(a) Stress versus plastic strain

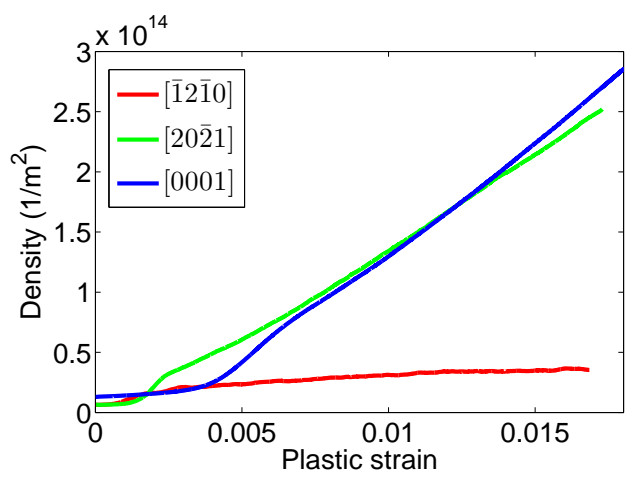

(b) Total dislocation density versus plastic strain

Fig. 7. (a) Stress versus plastic strain relationship and (b) corresponding total dislocation density for the three bulk beryllium runs: simple shear along the [12 $2 \overline{1} 0]$, tension loading along [2021], and [0001] directions.

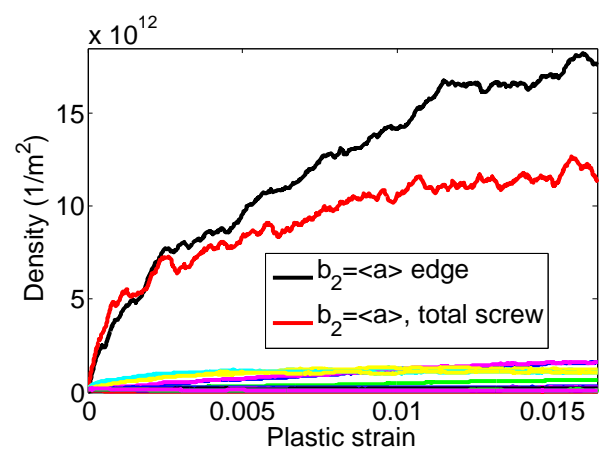

(a) Density per Burgers vector

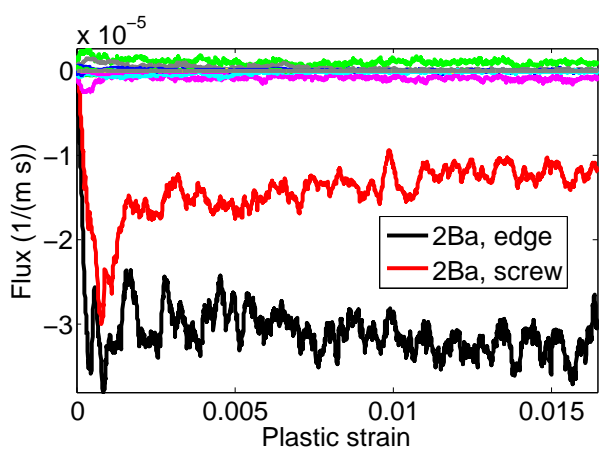

(b) Flux per slip system

Fig. 8. Density per Burgers vectors and flux per slip system for the bulk simulation of beryllium for a simple shear along the [12 $2 \overline{1} 0]$ direction. (a) Density per Burgers vector. (b) Flux per slip system. The main active slip system is $2 \mathrm{Ba}$. The other slip systems are shown on the figures but not labeled as their contribution is negligible.

More hardening is observed in the simulation of uniaxial straining along [20ㄹ1], Fig. 7. As expected from the resolved glide stresses and confirmed by the nonzero fluxes in Fig. 9[a]-[b], the two most active slip systems are 1PyII and $3 \mathrm{PyII}$ followed by $1 \mathrm{Ba}$ and $3 \mathrm{Ba}$. The same slip systems develop the highest dislocation densities, as shown in Fig. 10[a]-[b]. Interestingly, two slip systems with zero resolved glide stress, namely $2 \mathrm{Ba}$ and $2 \mathrm{PyII}$, show substantial dislocation density increase (Fig. 10[c]) but their associated dislocation fluxes remain near zero (Fig. 9[c]). More detailed analysis of the same simulation reveals that dislocations of Burgers vector $\boldsymbol{b}_{\mathbf{2}}$ in the $2 \mathrm{Ba}$ and $2 \mathrm{PyII}$ systems result from junctions generated by intersecting dislocations $\boldsymbol{b}_{\mathbf{1}}$ and $\boldsymbol{b}_{\mathbf{3}}$ moving on their respective glide planes. As shown in Figs. 13, the density of $\boldsymbol{b}_{\mathbf{2}}$ dislocations grows to a about half of the densities of $\boldsymbol{b}_{\mathbf{1}}$ and $\boldsymbol{b}_{\mathbf{3}}$ dislocations even though Burgers vector $\boldsymbol{b}_{\mathbf{2}}$ is perpendicular to the load direction. The $\boldsymbol{b}_{\mathbf{2}}$ junctions, once formed, remain largely stationary while acting as obstacles to continued glide of $\boldsymbol{b}_{\mathbf{1}}$ and $\boldsymbol{b}_{\mathbf{3}}$ dislocations. 


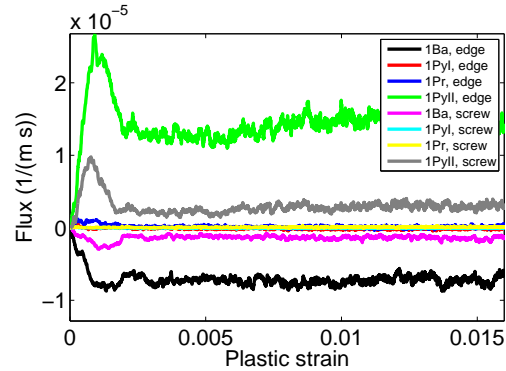

(a) Flux per slip system for $\boldsymbol{b}_{\mathbf{1}}=\langle a\rangle$

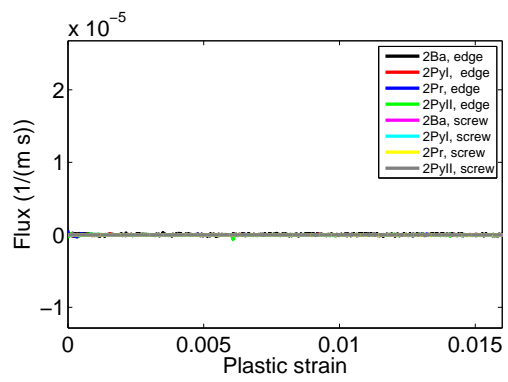

(c) Flux per slip system for $\boldsymbol{b}_{\mathbf{2}}=\langle a\rangle$

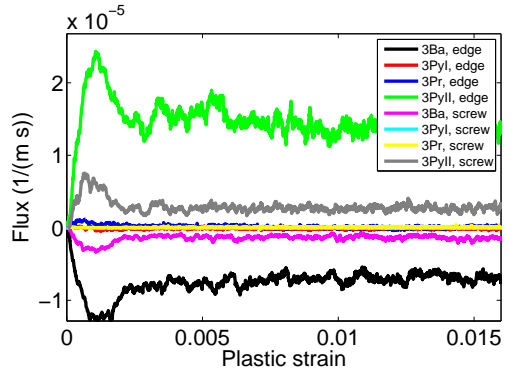

(b) Flux per slip system for $\boldsymbol{b}_{3}=<a>$

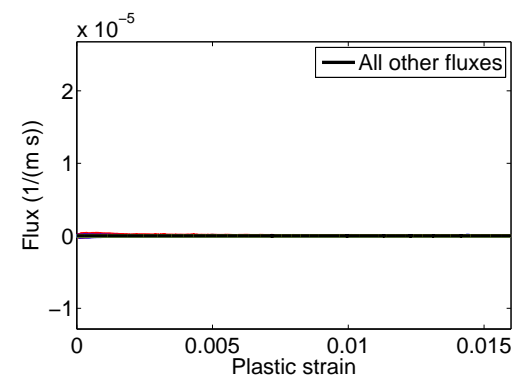

(d) Flux for non- $<$ a $>$ slip system

Fig. 9. Flux per slip system for the bulk simulation of beryllium subjected tension in the [2021] direction. (a) Fluxes for the Burgers vector $\boldsymbol{b}_{1}=\langle a\rangle$. (b) Fluxes for the Burgers vector $\boldsymbol{b}_{3}=<a>$. (c) Fluxes associated with Burgers vector $\boldsymbol{b}_{\mathbf{2}}$. (d) Fluxes for all the remaining slip systems.

The most strain hardening is observed in our third simulation of tension along [0001] axis, Fig. 7. As can be seen in Fig. 11, the three $<c+a>$ slip systems on the second pyramidal planes that see the highest resolved shear stress contribute most to the net plasticity of the crystal. Three slip systems of the same $<\mathrm{c}+\mathrm{a}\rangle$ dislocations on the first pyramidal planes are second most active for this loading direction. Fig. 12 shows densities of $\langle a\rangle,\langle\mathrm{c}\rangle$ and $<\mathrm{c}+\mathrm{a}>$ dislocations resolved by slip system (by symmetry, only one figure for each Burgers vector is shown). The three most active $<\mathrm{c}+\mathrm{a}>$ slip systems are largely responsible for a marked increase of $\langle\mathrm{c}+\mathrm{a}\rangle$ density by multiplication. Densities of $\langle\mathrm{a}\rangle$ and $\langle\mathrm{c}\rangle$ dislocation are also seen to rise concomitant with the increasing strain (Fig. 12[a]-[b]), but their associated flux components remain near zero (Fig. 11[b]). A detailed analysis of dislocation trajectories confirms that additional $\langle\mathrm{a}\rangle$ and $\langle\mathrm{c}\rangle$ dislocations are formed primarily by reactions among active $<\mathrm{c}+\mathrm{a}>$ dislocations.

As illustrated in Fig. 10[c] and Figs. 12[a]-[b], in strained HCP crystals a significant fraction of new dislocation density can be generated in various reactions of junction formation. While not necessarily active in producing plastic strain, the junction dislocations may contribute to forest hardening acting as obstacles to mobile dislocations. 


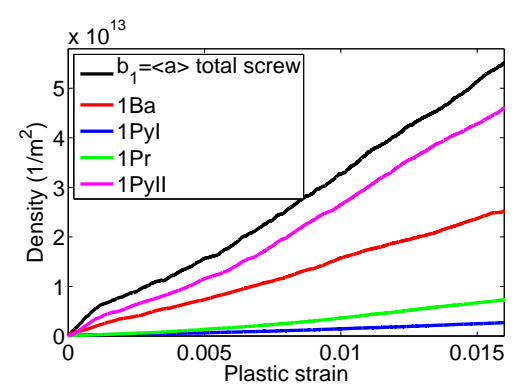

(a) Density for Burgers vector $\boldsymbol{b}_{\mathbf{1}}=\langle a\rangle$

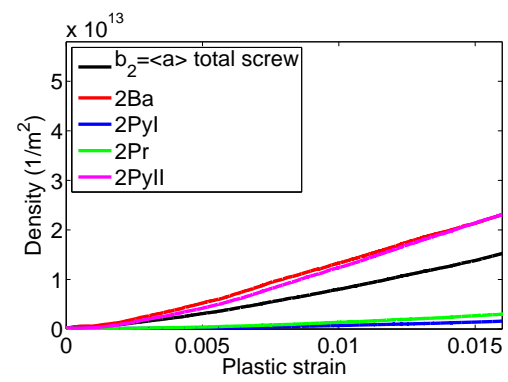

(c) Density for Burgers vector $\boldsymbol{b}_{2}=\langle a\rangle$

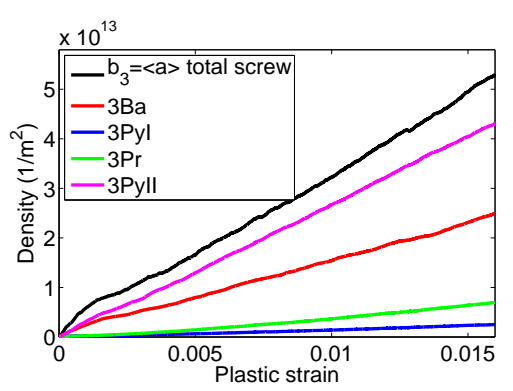

(b) Density for Burgers vector $b_{3}=<a>$

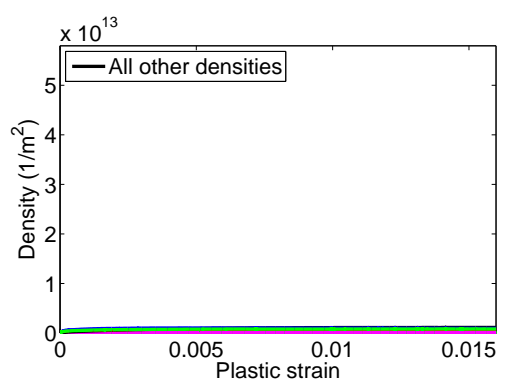

(d) Density for non- $<$ a $>$ Burgers vectors

Fig. 10. Density per Burgers vector for the bulk simulation of beryllium subjected tension in the [20211] direction. (a) Densities of Burgers vectors of type $\boldsymbol{b}_{1}=\langle a\rangle$. (b) Densities of Burgers vectors of type $\boldsymbol{b}_{3}=\langle a\rangle$. (c) Densities of Burgers vectors of type $\boldsymbol{b}_{2}=\langle a\rangle$. (d) Densities of Burgers vector of all other Burgers vector types.

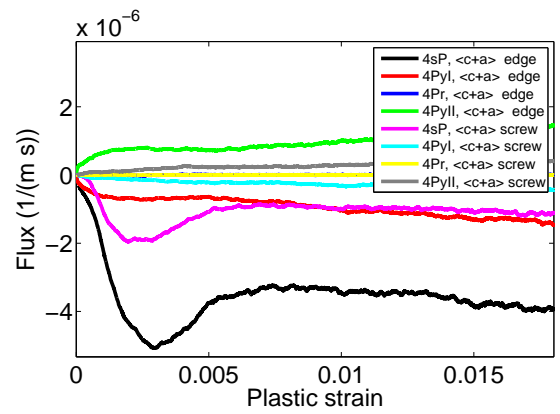

(a) Flux for $\boldsymbol{b}_{\mathbf{4}}=\langle c+a\rangle$

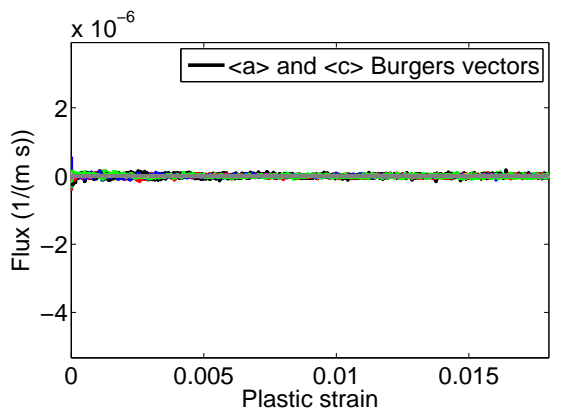

(b) Flux for $<a>$ and $<\mathrm{c}>$ slip systems

Fig. 11. Flux per slip system for the bulk simulation of beryllium subjected tension in the [0001] direction. (a-f) Flux for the $<\mathrm{c}+\mathrm{a}>$ Burgers vectors. (g) Flux for the non- $<\mathrm{c}+\mathrm{a}>$ Burgers vectors.

Finally, the effect of splintering $<\mathrm{c}+\mathrm{a}>$ dislocations is shown in Figs. 14- 16 . Figs. 14 compare stress/strain response and density evolution when composite dislocations of type $\langle c+a\rangle$ are allowed to split into $\langle c\rangle$ and $\langle a\rangle$ type dislocations and when they are not. The overall effect of the splintering of $\langle\mathrm{c}+\mathrm{a}\rangle$ dislocations is not negligible, it increases the overall dislocation density as well as yield and flow stress. 


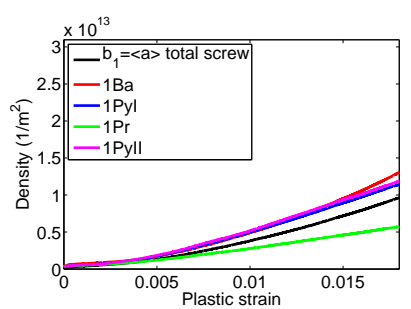

(a) Density for Burgers vector $\boldsymbol{b}_{\mathbf{1}}=\langle a\rangle$

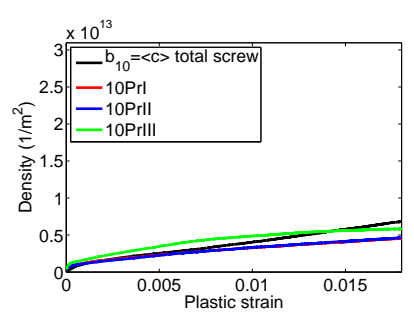

(b) Density for Burgers vector $\boldsymbol{b}_{10}=\langle c\rangle$

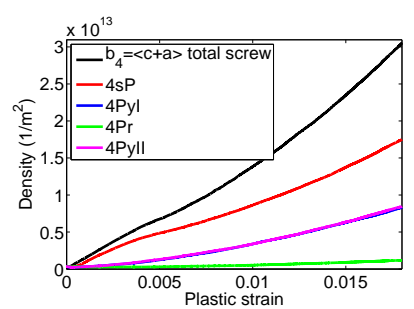

(c) Density for Burgers vector $\boldsymbol{b}_{\mathbf{4}}=\langle c+a\rangle$

Fig. 12. Density per Burgers vector for the bulk simulation of beryllium subjected tension in the [0001] direction. (a) Density associated with Burgers vectors of type $<$ a $>$. By symmetry, $\boldsymbol{b}_{\mathbf{1}}, \boldsymbol{b}_{\mathbf{2}}$, and $\boldsymbol{b}_{\mathbf{3}}$, show the same densities, only $\boldsymbol{b}_{\mathbf{1}}$ is shown. (b) Density for the slip systems associated with the Burgers vector of type $<c>$. (c) Density for the $<\mathrm{c}+\mathrm{a}>$ type Burgers vector. By symmetry, only the Burgers vector $\boldsymbol{b}_{\boldsymbol{4}}$ is shown. Burgers vectors of type $<\mathrm{c}+\mathrm{a}>$, from $\boldsymbol{b}_{\mathbf{4}}$ to $\boldsymbol{b}_{\mathbf{9}}$, show the same density profile.

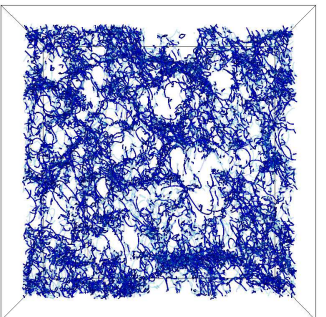

(a) Burgers vector or type $\boldsymbol{b}_{\mathbf{1}}=<a>$

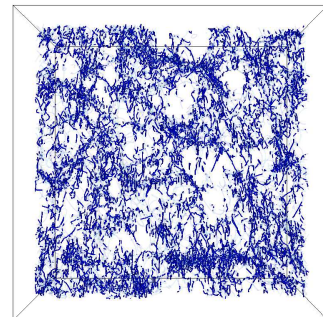

(b) Burgers vector oI type $\boldsymbol{b}_{\mathbf{2}}=\langle a\rangle$

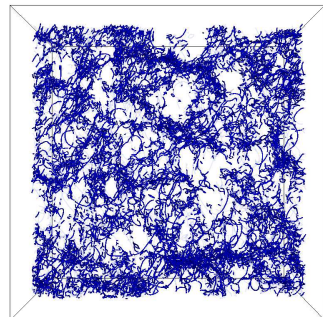

(c) Burgers vector of type $\boldsymbol{b}_{\mathbf{3}}=\langle a\rangle$

Fig. 13. Dislocation configurations obtained by dislocation dynamics simulations of beryllium for a loading along the [2021] direction showing only specific dislocation types: (a) dislocations with Burgers vector $\boldsymbol{b}_{1}$, (b) dislocations with Burgers vector $\boldsymbol{b}_{2}$, (c) dislocations with Burgers vector $\boldsymbol{b}_{3}$. The dislocations with Burgers vector $\boldsymbol{b}_{2}$ are numerous but do not move.

Figs. 15[a]-[b] show the ratio of the total dislocation density of $\langle a\rangle /<$ $c+a\rangle$ and $\langle c\rangle /\langle c+a\rangle$ respectively. These ratios increase when splintering is invoked. This is confirmed when looking at the dislocation network composed of Burgers vectors of type $\langle a\rangle$ only, Figs. 16[a-b] and of type $\langle c\rangle$ only, Figs. $16[\mathrm{c}-\mathrm{d}]$. For the same overall density, set to $2.96 \times 10^{14} \mathrm{~m}^{2}$ in Figs. 16, there are more dislocations of type $\langle a\rangle$ and $\langle c\rangle$ when splintering is allowed. Allowing dissociation of $\langle\mathrm{c}+\mathrm{a}\rangle$ dislocations changes the character of the dislocation density and leads to a higher hardening rate. The observed increase stiffness when composite dislocations of type $\langle c+a\rangle$ are allowed to dissociate may help explain the long-standing problem of low ductility of HCP materials and relate it to the density of $\langle c+a\rangle$ dislocations versus the density of $\langle c\rangle$ and $\langle a\rangle$ dislocations. 


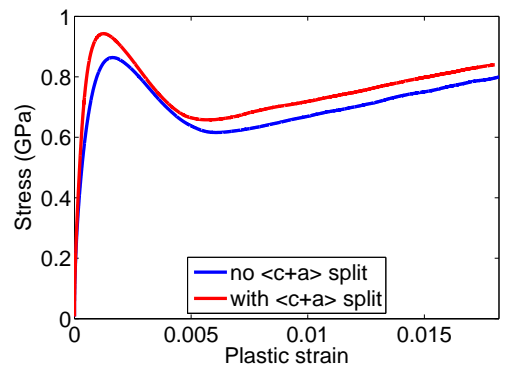

(a) Stress/Strain response

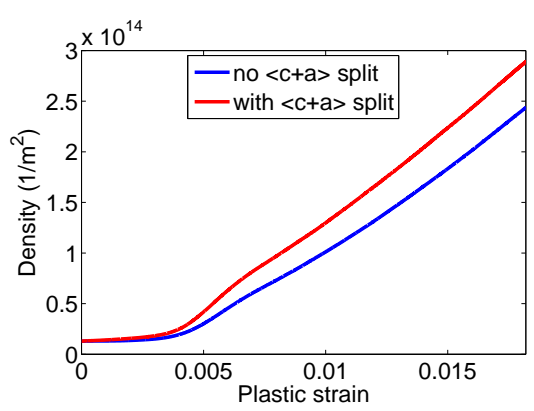

(b) Density evolution

Fig. 14. Effects of $\langle c+a>$ splintering on the (a) overall stress-strain response of beryllium materials, and (b) total dislocation density.

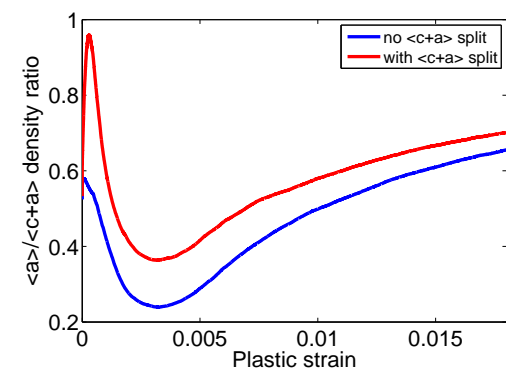

(a) Ratio between $\langle a\rangle /<$ $c+a>$ total density

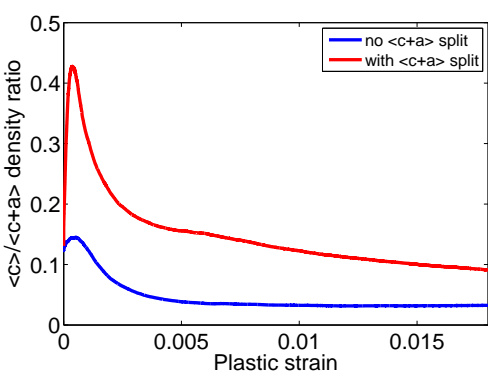

(b) Ratio between $\langle c\rangle /<$ $c+a>$ total density

Fig. 15. Density ratio showing the effects of $<\mathrm{c}+\mathrm{a}>$ splintering on large scale beryllium simulations.

\section{Summary and conclusions}

The slip plasticity of HCP crystals involves dislocations of three distinct types of Burgers vectors that can move on a variety of glide planes. One interesting consequence of the HCP slip crystallography is the appearance of composite dislocations, including $<\mathrm{c}+\mathrm{a}>$ type, $<2 \mathrm{a}>$ type, and $<\mathrm{d}>$ type dislocations. Under certain conditions, dissociation of such composite dislocations can give rise to rather unusual modes of cross-slip where, following a dissociation event, each of the two component dislocations can glide away on their own glide plane from a composite parent that was initially non-screw. Additionally, dissociation of composite dislocations formed by binary reactions can impart additional strength to dislocation junctions compared to their undissociated configuration and may have appreciable effect on HCP strength and strain hardening.

To enable accurate DD simulations of HCP materials, we developed several practical methods to account for dislocation mechanisms not present in published DD algorithms of cubic crystals. A novel interpolation scheme was presented that approximates mobility of a mixed dislocation from the mobilities of pure screw and edge dislocations. The scheme is based on geometric parti- 


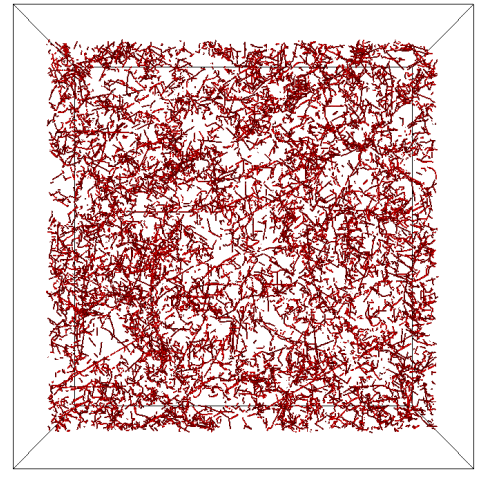

(a) $<a>$ density with no splintering

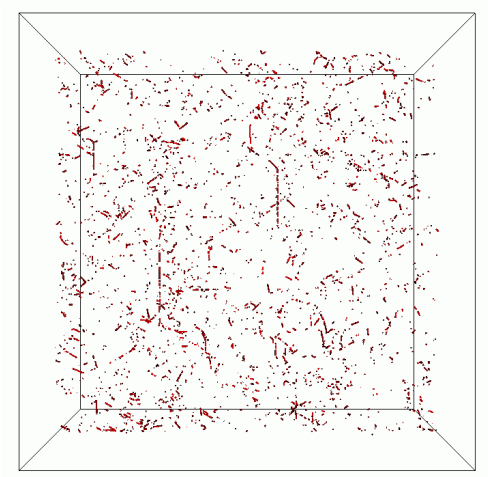

(c) $\langle c>$ density with no splintering

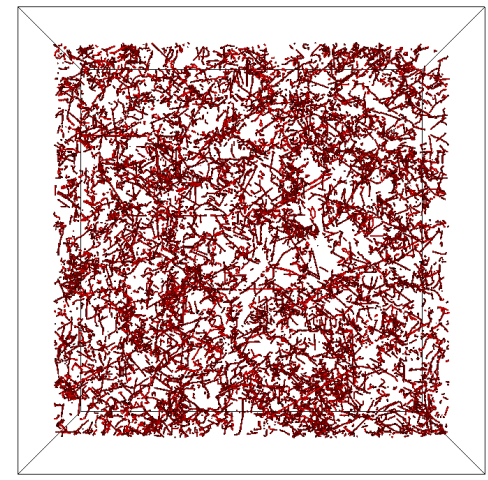

(b) $<a>$ density with splintering

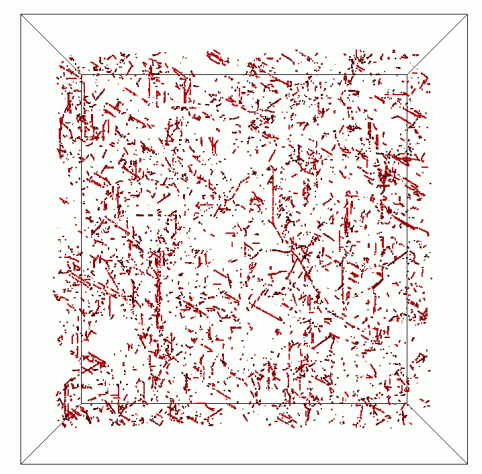

(d) $<c>$ density with splintering

Fig. 16. Dislocation density for $\langle a\rangle$ and $\langle c\rangle$ Burgers vectors for the same overall density with and without the splintering topological operations. More density is observed when splintering is allowed.

tioning of the dislocation line orientation (character) into its edge and screw components and assembly a mixed mobility from known mobilities of the two pure components. The interpolation procedure is not specific to HCP crystals, may be applicable to both linear and non-linear mobilities, and can take into account the mobility information for a number of dislocation orientations seamlessly.

Finally, DD simulations of bulk plasticity in HCP beryllium under three different straining conditions were conducted. Perhaps the most noteworthy observation from our bulk DD simulations is that large numbers of stationary dislocations are generated via junction reactions between mobile $<\mathrm{a}>$ and $<\mathrm{c}+\mathrm{a}>$ dislocations. Even when stationary, such junction products should not be neglected because they are appreciable in density, and they may act as 
strong obstacles to other mobile dislocations in the dislocation network. Furthermore, their motion was limited in the simulations conducted, not by an inherent immobility, but by the loading directions chosen. Other loading directions may lead to a more complex co-evolution of $\langle\mathrm{a}\rangle,\langle\mathrm{c}\rangle$, and $\langle\mathrm{c}+\mathrm{a}\rangle$ type Burgers vectors. Our DD simulation data was used to analyze the evolution of dislocation density and flux partitioned over individual Burgers vectors and slip systems. In addition to revealing how the different components of plastic slip interact, the same data provides coarse-grained measures of dislocation plasticity that may be valuable in the construction of continuum crystal plasticity models of type reported in [4]. 


\section{Appendix}

\section{A Decomposition of a dislocation line segment and density per Burgers vector}

A general dislocation line geometry is described by its Burgers vector and its line direction. The Burgers vectors in crystals are discrete but the line directions are continuous. For convenience and connection to dislocation densitybased continuum plasticity models, it may be advantageous to decompose a general dislocation line segment into $n$ components as follows

$$
\boldsymbol{\ell}_{\boldsymbol{D} \boldsymbol{D}}=\sum_{i=1}^{n} \ell_{i} \boldsymbol{t}_{i}, \text { where } \ell_{i} \text { satisfy } \min _{\ell_{i}}\left[\sum_{i=1}^{n}\left|\ell_{i}\right|\right] \text {. }
$$

where $\boldsymbol{\ell}_{\boldsymbol{D} \boldsymbol{D}}=\boldsymbol{p}_{2}-\boldsymbol{p}_{1}$ is a dislocation line segment with Burgers vector $\boldsymbol{b}$, the directions $\boldsymbol{t}_{i}, i=1, \ldots n$, are pre-defined, and the $\ell_{i}$ are unknowns that satisfy the optimality condition. Finding the length components solution of Eq. A.1 is a classic linear programming problem.

For instance, the set of directions $\boldsymbol{t}_{i}$ can be associated to screw and edge directions of the Burgers vector of the dislocation line. In BCC crystals, there is a set of four Burgers vectors and three glide planes, this means that a line direction in this crystal can be decomposed into a screw and three edges components. Similarly, in FCC crystals, the line direction can be decomposed into a screw component and two edge components. In HCP crystals, the line direction can be decomposed into a screw and four edge components.

Another example is to choose important directions identified by properties of the crystal such as $60^{\circ}$ and $120^{\circ}$ line directions for hcp crystals.

The dislocation densities are then defined by

$$
\rho^{i}=\frac{\left|\ell^{i}\right|}{V}, i=1, \ldots, n
$$

where $V$ is the volume of the simulation.

\section{B Decomposition of the plastic strain and dislocation flux per slip system}

In the DD method, the plastic strain rate contribution associated with each dislocation segment is calculated by the area swept by the segment per unit 
time increment. In general, however the line segment at the end of the time increment is not coplanar with the line segment due to the flexibility of the DD code. The plastic strain rate tensor, $\dot{\boldsymbol{\epsilon}}_{p}$, associated with a single dislocation line segment is given by

$$
\dot{\boldsymbol{\epsilon}}_{p}=\frac{1}{2 \Delta t} \frac{1}{V} \operatorname{sym}\left[\boldsymbol{b} \otimes\left(\boldsymbol{\ell}_{\boldsymbol{D} \boldsymbol{D}}(t) \times \boldsymbol{\Delta} \boldsymbol{x}_{\mathbf{1}}+\boldsymbol{\ell}_{\boldsymbol{D} \boldsymbol{D}}(t+\Delta t) \times \boldsymbol{\Delta} \boldsymbol{x}_{\mathbf{2}}\right)\right]
$$

where $\boldsymbol{b}$ is the Burgers vector, $\boldsymbol{\ell}_{\boldsymbol{D} \boldsymbol{D}}(t)=\boldsymbol{p}_{2}(t)-\boldsymbol{p}_{1}(t)$ is the line segment at time $t, \boldsymbol{\Delta} \boldsymbol{x}_{\mathbf{1}}=\boldsymbol{p}_{1}(t+\Delta t)-\boldsymbol{p}_{1}(t)$ is the change in the position of the first node of the segment, and $\boldsymbol{\Delta} \boldsymbol{x}_{\mathbf{2}}=\boldsymbol{p}_{2}(t+\Delta t)-\boldsymbol{p}_{2}(t)$ is the change in the position of the second node of the segment during an interval of time $\Delta t$.

There is an equivalent description that can be written in terms of the dislocation density fluxes and uses the definition of the dislocation line segment in Eq. A.1

$$
\dot{\boldsymbol{\epsilon}}_{p}=\operatorname{sym}\left[\boldsymbol{b} \otimes\left(\sum_{i=1}^{n} q_{i} \boldsymbol{n}_{i}+\sum_{i=1}^{n} q_{i}^{s} \boldsymbol{n}_{i}^{s}+q^{\text {non-glide }} \frac{\boldsymbol{b}}{|\boldsymbol{b}|}\right)\right]
$$

where $q_{i}$ is the flux of dislocations on plane $\boldsymbol{n}_{i}=\frac{\boldsymbol{t}_{i} \times \boldsymbol{b}}{\left|\boldsymbol{t}_{i} \times \boldsymbol{b}\right|}$, where the $\boldsymbol{t}_{i}$ are the pre-defined directions described in Eq. A.1. $q^{\text {non-glide }}$ is the flux for non-glide dislocations along $\frac{\boldsymbol{b}}{|\boldsymbol{b}|}$, and $q_{i}^{s}$ represents the flux of dislocations for which $\boldsymbol{b}$ is parallel to $\boldsymbol{t}_{i}$, and is given for $\boldsymbol{n}_{i}^{s}$ pre-defined planes directions. The planes $\boldsymbol{n}_{i}^{s}$ can be chosen to be the same as the $\boldsymbol{n}_{i}$ planes. The fluxes $q_{\text {non-glide }}, q_{i}$, and $q_{i}^{s}$ for $i=1, \ldots, n$ are the unknowns to be found.

Using the decomposition of the dislocation line segment Eq. A.1 and inserting it in Eq. B.1, the fluxes solution of Eq. B.2 are

$$
\begin{gathered}
q_{i}=\frac{1}{2 \Delta t}\left[\ell_{i}(t) \boldsymbol{t}_{i} \times \boldsymbol{\Delta} \boldsymbol{x}_{\mathbf{1}}+\ell_{i}(t+\Delta t) \boldsymbol{t}_{i} \times \boldsymbol{\Delta} \boldsymbol{x}_{\mathbf{2}}\right] \cdot \boldsymbol{n}_{i} \\
q^{\text {non-glide }}=\frac{1}{2 \Delta t}\left[\boldsymbol{\ell}_{\boldsymbol{D} \boldsymbol{D}} \times \boldsymbol{\Delta} \boldsymbol{x}_{\mathbf{1}}+\boldsymbol{\ell}_{\boldsymbol{D} \boldsymbol{D}}(t+\Delta t) \times \boldsymbol{\Delta} \boldsymbol{x}_{\mathbf{2}}\right] \cdot \frac{\boldsymbol{b}}{|\boldsymbol{b}|}
\end{gathered}
$$

For the fluxes $q_{i}^{s}, i=1, \ldots, n$, a system of equations as was solved in the dislocation density case needs to be defined. If

$$
q_{i}^{s}=\frac{1}{2 \Delta t}\left[\ell_{i}(t) \boldsymbol{t}_{i} \times \boldsymbol{\Delta} \boldsymbol{x}_{\mathbf{1}}+\ell_{i}(t+\Delta t) \boldsymbol{t}_{i} \times \boldsymbol{\Delta} \boldsymbol{x}_{\mathbf{2}}\right] \cdot \boldsymbol{n}_{i}^{s}
$$

then adding the optimality condition

$$
\min _{q_{s}^{i}}\left[\sum_{i=1}^{n}\left|q_{i}^{s}\right|\right],
$$

is sufficient to solve for the fluxes $q_{i}^{s}$, for $i=1, \ldots, n$. 


\section{Acknowledgments}

This article is dedicated to the memory of Gregg Hommes.

This work performed under the auspices of the U.S. Department of Energy by Lawrence Livermore National Laboratory under Contract DE-AC52-07NA27344. Also, research was sponsored by the Army Research Laboratory and was accomplished under Cooperative Agreement Number W911NF-12-2-0022. The views and conclusions contained in this document are those of the authors and should not be interpreted as representing the official policies, either expressed or implied, of the Army Research Laboratory or the U.S. Government. The U.S. Government is authorized to reproduce and distribute reprints for Government purposes notwithstanding any copyright notation herein.

\section{References}

[1] S. Groh, E. B. Marin, M. F. Horstemeyer, and D. J. Bammann. Dislocation motion in magnesium: a study by molecular statics and molecular dynamics. Modelling and Simulation in Materials Science and Engineering, 17(7):075009, 2009 .

[2] R.R. Boyer. An overview on the use of titanium in the aerospace industry. Materials Science and Engineering: A, 213(12):103 - 114, 1996. International Symposium on Metallurgy and Technology of Titanium Alloys.

[3] A. Arsenlis, W. Cai, M. Tang, M. Rhee, T. Oppelstrup, G. Hommes, T.G. Pierce, and V. V. Bulatov. Enabling strain hardening simulations with dislocation dynamics. Modelling and Simulation in Materials Science and Engineering, 15(6):553-595, 2007.

[4] N. R. Barton, J. V. Bernier, R. Becker, A. Arsenlis, R. Cavallo, J. Marian, M. Rhee, H.-S. Park, B. A. Remington, and R. T. Olson. A multiscale strength model for extreme loading conditions. Journal of Applied Physics, 109(7):073501, 2011.

[5] M. H. Yoo and J. K. Lee. Deformation twinning in h.c.p. metals and alloys. Philosophical Magazine A, 63(5):987-1000, 1991.

[6] H. Fan, S. Aubry, A. Arsenlis, and J. A. El-Awady. Discrete dislocation dynamics simulations of twin size-effects in magnesium. In Mater. Res. Soc. Symp. Proc., volume 1741. Materials Research Society, 2015.

[7] H. Fan, S. Aubry, A. Arsenlis, and J. El Awady. Grain size effects on dislocation and twinning mediated plasticity in magnesium. Scripta Mater., 112:50-53, 2016 . 
[8] H. Fan, S. Aubry, A. Arsenlis, and J. El Awady. The role of twinning deformation on the hardening response of polycrystalline magnesium from discrete dislocation dynamics simulations. Acta Mater, 92:126-139, 2015.

[9] H. Fan, S. Aubry, A. Arsenlis, and J. El Awady. Orientation influence on grain size effects in ultrafine-grained magnesium. Scripta Mater., 97:25-28, 2015.

[10] L Capolungo, IJ Beyerlein, and Z Qwang. The role of elastic anisotropy on plasticity in hcp metals: a three-dimensional dislocation dynamics study. Modelling and Simulation in Materials Science and Engineering, 18(8):085002, 2010.

[11] G. Monnet, B. Devincre, and L. P. Kubin. Dislocation study of prismatic slip systems and their interactions in hexagonal close packed metals: application to zirconium. Acta Materialia, 52(14):4317 - 4328, 2004.

[12] L. Capolungo and I. J. Beyerlein. Nucleation and stability of twins in hcp metals. Phys. Rev. B, 78:024117, Jul 2008.

[13] N. Bertin, I.J. Beyerlein C.N. Tome, M.R. Barnett, and L. Capolungo. On the strength of dislocation interactions and their effect on latent hardening in pure magnesium. International Journal of Plasticity, 62:pp. 72-92, 2014.

[14] S.R. Agnew, J.A. Horton, and Yoo M.H. Transmission electron microscopy investigation of $<\mathrm{c}+\mathrm{a}>$ dislocations in $\mathrm{mg}$ and $\alpha$-solids solution mg-li alloys. Metallurgical and materials transactions A, 33A:851, 2002.

[15] Z. Wu and Curtin W.A. The origins of high hardening and low ductility in magnesium. Nature, 526:62-67, 2015.

[16] M.H. Yoo, S.R. Agnew, J.R. Morris, and K.M. Hob. Non-basal slip systems in hcp metals and alloys: source mechanisms. Materials Science and Engineering: A, 319-321(0):87 - 92, 2001.

[17] M.H. Yoo. c+a dislocations reactions in h.c.p. metals. Scripta Metallurgica, 2:537-540, 1968.

[18] Hideki Tonda and Shinji Ando. Effect of temperature and shear direction on yield stress by $\{11 \overline{2} 2\}[\overline{1} \overline{1} 23]$ slip in hcp metals. Metallurgical and Materials Transactions A, 33(3):831-836, 2002.

[19] Damiano V. V. The double tetrahedron - a method of notation for cph structures. Transactions of the metallurgical society of AIME, 227:788-789, 1963.

[20] D. Hull and Bacon D. J. Introduction to Dislocations. Elsevier, 2001.

[21] V. V. Bulatov and W. Cai. Computer Simulations of Dislocations. Oxford University Press, 2006.

[22] S. Aubry and A. Arsenlis. Use of spherical harmonics for dislocation dynamics in anisotropic elastic media. Modelling and Simulation in Materials Science and Engineering, 21(6):065013, 2013. 
[23] S. Aubry, S.P. Fitzgerald, and A. Arsenlis. Methods to compute dislocation line tension energy and force in anisotropic elasticity. Modelling and Simulation in Materials Science and Engineering, 22(1):015001, 2014.

[24] W. Cai and V. Bulatov. Mobility laws in dislocation dynamics simulations. Mat. Sci. and Eng. A, 387-389:277-281, 2004.

[25] M.R. Gilbert, S. Queyreau, and J. Marian. Stress and temperature dependence of screw dislocation mobility in $\alpha$-fe by molecular dynamics. Physical Review B, 84(17):174103, 2011.

[26] S. Queyreau, G. Monnet, and B. Devincre. Orowan strengthening and forest hardening superposition examined by dislocation dynamics simulations. Acta Materialia, 58(17):5586 - 5595, 2010.

[27] Fritz Aldinger. Flow and fracture of single crystals(beryllium crystal dislocations). Beryllium science and technology, 1:7-114, 1979.

[28] V. V. Bulatov, L. L. Hsiung, M. Tang, A. Arsenlis, W. Cai M. C. Bartelt, J. N. Florando, M. Hiratani, M. Rhee, G. Hommes, T. G. Pierce, and T. Diaz de la Rubia. Dislocation multi-junctions and strain hardening. Nature, 440:11741178, 2006.

[29] F. Kroupa. The force between non-parallel dislocations. Czech J. Phys., B 11:847-848, 1961.

[30] J.P. Hirth and J. Lothe. Theory of Dislocations. Krieger publishing company, 1982.

[31] H. Ledbetter and A. Migliori. A general elastic-anisotropy measure. Journal of Applied Physics, 100:063516, 2006.

[32] D. Tromans. Elastic anisotropy of hcp metals crystals and polycrystals. International Journal of Research and Reviews in Applied Sciences, 6(4):462483, 2011.

[33] C-C. Wu, S. Aubry, P. W. Chung, and A. Arsenlis. Dislocation dynamics simulations of junctions in hexagonal close-packed crystals. MRS Proceedings, Symposium SS:1424, 2012.

[34] C-C. Wu, P. W. Chung, S. Aubry, and A. Arsenlis. Dislocation dynamics simulations of junctions in hexagonal close-packed crystals. Acta Materialia, 61:3422-3431, 2013. 